\title{
Comparison of methods to detect the in vitro activity of silver nanoparticles (AgNP) against multidrug resistant bacteria
}

Emerson Danguy Cavassin', Luiz Francisco Poli de Figueiredo², José Pinhata Otoch², Marcelo Martins Seckler ${ }^{3}$, Roberto Angelo de Oliveira ${ }^{3}$, Fabiane Fantinelli Franco ${ }^{4}$, Valeria Spolon Marangoni ${ }^{4}$, Valtencir Zucolotto ${ }^{4}$, Anna Sara Shafferman Levin ${ }^{1}$ and Silvia Figueiredo Costa ${ }^{1,5^{*}}$

\begin{abstract}
Background: Multidrug resistant microorganisms are a growing challenge and new substances that can be useful to treat infections due to these microorganisms are needed. Silver nanoparticle may be a future option for treatment of these infections, however, the methods described in vitro to evaluate the inhibitory effect are controversial.

Results: This study evaluated the in vitro activity of silver nanoparticles against 36 susceptible and 54 multidrug resistant Gram-positive and Gram-negative bacteria from clinical sources. The multidrug resistant bacteria were oxacilin-resistant Staphylococcus aureus, vancomycin-resistant Enterococcus spp., carbapenem- and polymyxin B-resistant A. baumannii, carbapenem-resistant $P$. aeruginosa and carbapenem-resistant Enterobacteriaceae. We analyzed silver nanoparticles stabilized with citrate, chitosan and polyvinyl alcohol and commercial silver nanoparticle. Silver sulfadiazine and silver nitrate were used as control. Different methods were used: agar diffusion, minimum inhibitory concentration, minimum bactericidal concentration and time-kill. The activity of AgNPs using diffusion in solid media and the MIC methods showed similar effect against MDR and antimicrobial-susceptible isolates, with a higher effect against Gram-negative isolates. The better results were achieved with citrate and chitosan silver nanoparticle, both with $\mathrm{MIC}_{90}$ of $6.75 \mu \mathrm{g} \mathrm{mL} \mathrm{L}^{-1}$, which can be due the lower stability of these particles and, consequently, release of $\mathrm{Ag}^{+}$ions as revealed by X-ray diffraction (XRD). The bactericidal effect was higher against antimicrobial-susceptible bacteria.

Conclusion: It seems that agar diffusion method can be used as screening test, minimum inhibitory concentration/ minimum bactericidal concentration and time kill showed to be useful methods. The activity of commercial silver nanoparticle and silver controls did not exceed the activity of the citrate and chitosan silver nanoparticles. The in vitro inhibitory effect was stronger against Gram-negative than Gram-positive, and similar against multidrug resistant and susceptible bacteria, with best result achieved using citrate and chitosan silver nanoparticles. The bactericidal effect of silver nanoparticle may, in the future, be translated into important therapeutic and clinical options, especially considering the shortage of new antimicrobials against the emerging antimicrobial resistant microorganisms, in particular against Gram-negative bacteria.
\end{abstract}

Keywords: Silver nanoparticle, Antimicrobial test, Multidrug resistant bacteria

\footnotetext{
*Correspondence: silviacosta@usp.br; costasilviaf@ig.com.br

${ }^{5}$ LIM-54 (Laboratório de Bacteriologia), Instituto de Medicina Tropical,

Av. Dr. Enéas de Carvalho Aguiar, 470, Prédio II, $1^{\circ}$ andar, Sala 112,

Cerqueira César, São Paulo, SP 054503-00, Brazil

Full list of author information is available at the end of the article
} 


\section{Background}

In the last decade, the emergence of multidrug-resistant bacteria (MDR) became a challenge around the world [1]. Antimicrobial resistance is recognized as a medical problem that increases mortality, morbidity rates, length of stay and cost and has nowadays few therapeutic possibilities [2]. In this scenario, new products such as nanotechnology may have a role to treat infections due to MDR [3].

Nanotechnology is opening possibilities, allowing new solutions with old resources. Nanoscale materials such as silver nanoparticles (AgNPs) have emerged as novel agents because their high surfaces area to volume ratio and the unique chemical and physical properties [4]. Silver nanoparticles have greater efficiency in mediating their antimicrobial activity when compared with silver salts $[5,6]$. Because AgNPs acts synergistically in distinct targets it is expected that there will be no interference with antimicrobial resistance mechanisms. Thus, AgNPs has a potential use to treat MDR.

However, the mechanisms by which AgNPs act against bacteria are not yet fully elucidated. It is believed that in aqueous solution biologically active $\mathrm{Ag}^{+}$ions are delivered and promotes the antimicrobial effect [5-7]. Silver nanoparticles interact with three vital components of the cells: (a) peptidoglycan cell wall, (b) cytoplasmic membrane, where chemical and physical properties are modified and results in an imbalance of osmolality, permeability, electron transport, and cellular breathing and (c) ribosomal DNA, molecular sites of phosphorus and sulfur present in proteins, especially in enzymes involved in the electron transport chain [7].

Currently there is a recommendation by International Organization for Standardization (ISO), a worldwide federation of national standards $[8,9]$, for evaluating potency of silver nanoparticles against cell wall degradation of Staphylococcus aureus and muramic acid release, using gas chromatography-mass spectrometry (GC-MS) $[8,10]$.
However, studies in the literature have used the methods recommended for antimicrobial drugs: diffusion in agar and determination of minimum inhibitory concentration (MIC) [11-13] and minimum bactericidal concentration (MBC) according to the current Clinical and Laboratory Standards Institute (CLSI) document recommendation [14].

The aim of this study was to evaluate the in vitro activity of AgNPs stabilized with different compounds against MDR and antimicrobial-susceptible Gram-positive and Gram-negative microorganisms, using different methods.

\section{Results and discussion \\ AgNPs}

The size and morphology of the AgNPs were investigated by Field Emission Gun Scanning Electron MicroscopeFEG-SEM (Fig. 1). PVA-AgNPs and Chitosan-AgNPs presented an average diameter around 10 and $25 \mathrm{~nm}$, respectively, while the citrate-AgNPs have around $40 \mathrm{~nm}$ of diameter. The UV-VIS spectra in Fig. 2 show the optical properties of PVA, Chitosan and Citrate stabilized AgNPs. The results reveal the surface plasmon resonances peaks around $400 \mathrm{~nm}$ for all systems, which is typical for nanostructured silver [15-17]. It is well known that the surface plasmon resonances depend strongly on the size, shape and functionalization of the metallic NPs [18]. The increasing in the size of the AgNPs leads to a red-shift and broadening of the plasmon resonance band [18], which agree with the spectrum observed for CitrateAgNPs. The zeta potential analysis reveals significant differences in the surface charges between the three systems (Table 1). Chitosan AgNPs presented high positively surface charge $(+41.1 \mathrm{mV})$, while the citrate ones were very negative $(-48.4 \mathrm{mV})$ and PVA AgNPs were more close to zero $(-17.0 \mathrm{mV})$.

Figure 3 shows the $x$-ray diffraction spectra of the AgNPs stabilized by PVA, chitosan and citrate. The spectra of PVA AgNP and chitosan AgNP reveals the existence of the peaks at $2 \theta=38.15^{\circ}, 44.34^{\circ}, 64.5^{\circ}$ and $77.46^{\circ}$,
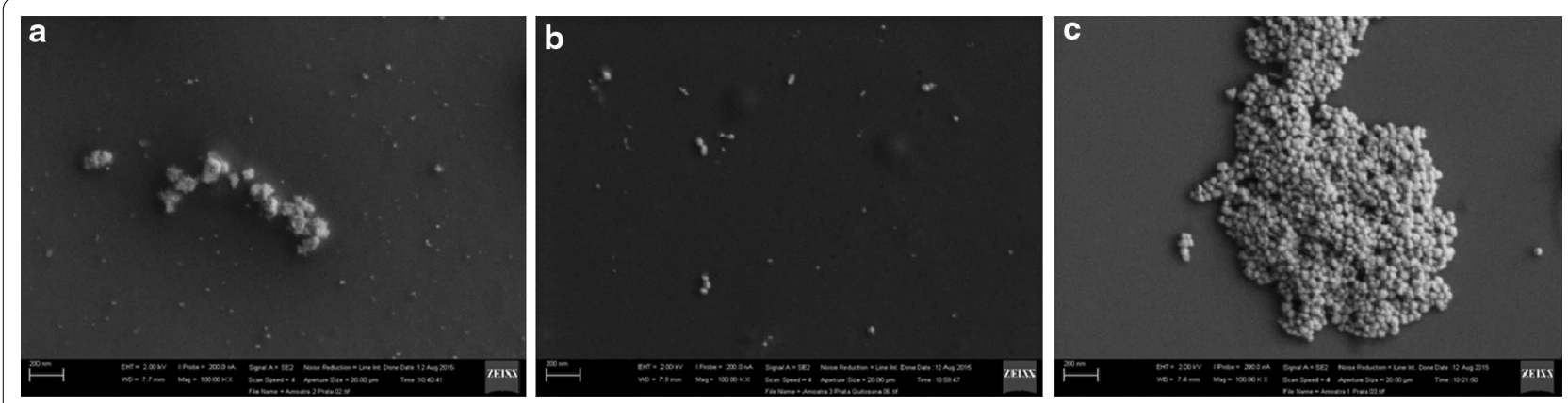

Fig. 1 FEG-SEM micrographs of AgNPs stabilized by a PVA, b chitosan and c citrate 


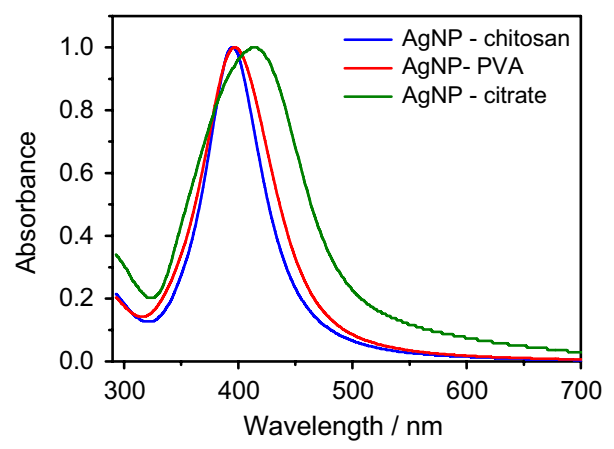

Fig. 2 UV-vis spectroscopy spectra of PVA, chitosan and citrate AgNPs

Table 1 Zeta potential for PVA, chitosan and citrate AgNPs

\begin{tabular}{ll}
\hline AgNPs & Potential zeta \\
\hline AgNP-PVA & $-17.0 \mathrm{mV}$ \\
AgNP-Chitosan & $+41.1 \mathrm{mV}$ \\
AgNP-Citrate & $-48.4 \mathrm{mV}$ \\
\hline
\end{tabular}

which can be assigned to the (111), (200), (220), and (311) reflections of the face centered cubic (fcc) structure of metallic silver, respectively [19-21]. No impurities were detected in the XRD profile of AgNPs-PVA. However, some intense peaks at $2 \theta$ angles of $32.2^{\circ}, 46.3^{\circ}, 54.7^{\circ}$ and $57.3^{\circ}$ were observed in the XRD profile of AgNPschitosan. Several studies attribute the presence of these small additional peaks to the crystalline organic phase $[22,23]$. On the other side, the peaks at $32.2^{\circ}$ and $54.7^{\circ}$ also match with the diffraction profile of the $\mathrm{Ag}_{2} \mathrm{O}[24$, 25 ] and might be an indicate that some silver oxide have been formed during the synthesis or the drying process of AgNPs in presence of chitosan.
The spectrum of citrate AgNPs shows no peak associate to metallic nanoparticle. The peak around $32^{\circ}$ can be can be assigned to silver salt. Since we observed the formation of the silver nanoparticles in all our previous characterizations such as FEG-SEM and UV-VIS spectroscopy, this finding suggests that the metallic silver were oxidized to ions during the drying process and implies that the sodium citrate is not a good stabilizer to protect the silver particles against oxidation.

The diameter of the particles (d) stabilized with PVA and chitosan were estimated by Scherrer equation (Eq. 1), where 0.94 is the constant value used for spherical particle shape, $\lambda$ is the X-ray wavelength $(0.15406 \mathrm{~nm}), B$ is the line broadening at half maximum intensity of the selected reflection plane in radians, and $\theta$ is the Bragg angle.

$$
d=\frac{0.94 \lambda}{B \cos \theta}
$$

The mean crystalline particles size were determined from the main $(111)$ diffraction peak $\left(2 \theta=38.15^{\circ}\right)$ of the $\mathrm{X}$-ray diffraction pattern. The diameter found for PVA was $7.1 \mathrm{~nm}$, which is close to the diameter found by SEM images. However, the calculated diameter for chitosanAgNPs were around $11.9 \mathrm{~nm}$, while the one obtained from the SEM images were $25 \mathrm{~nm}$. This difference might be related to the oxidation of silver ions during the drying process, which was showed in the X-ray pattern, and consequently decrease in the nanoparticle size.

\section{Growth inhibition by diffusion}

All inhibition zones produced were plotted according to their size (Fig. 4). The inhibition zone was similar comparing MDR and susceptible bacteria, with larger zones for citrate and chitosan AgNPs than PVA AgNPs. Silver nitrate had no activity against MDR by this method (Fig. 5).
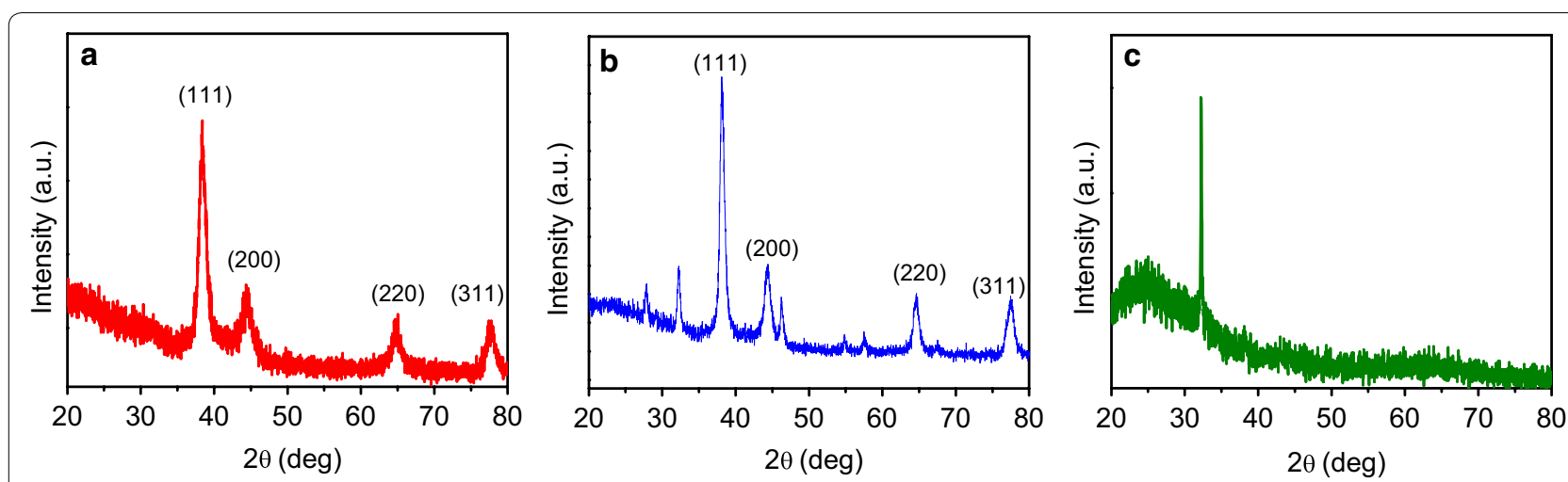

Fig. 3 X-ray diffraction patterns for AgNPs stabilized by a PVA, b chitosan and c citrate 

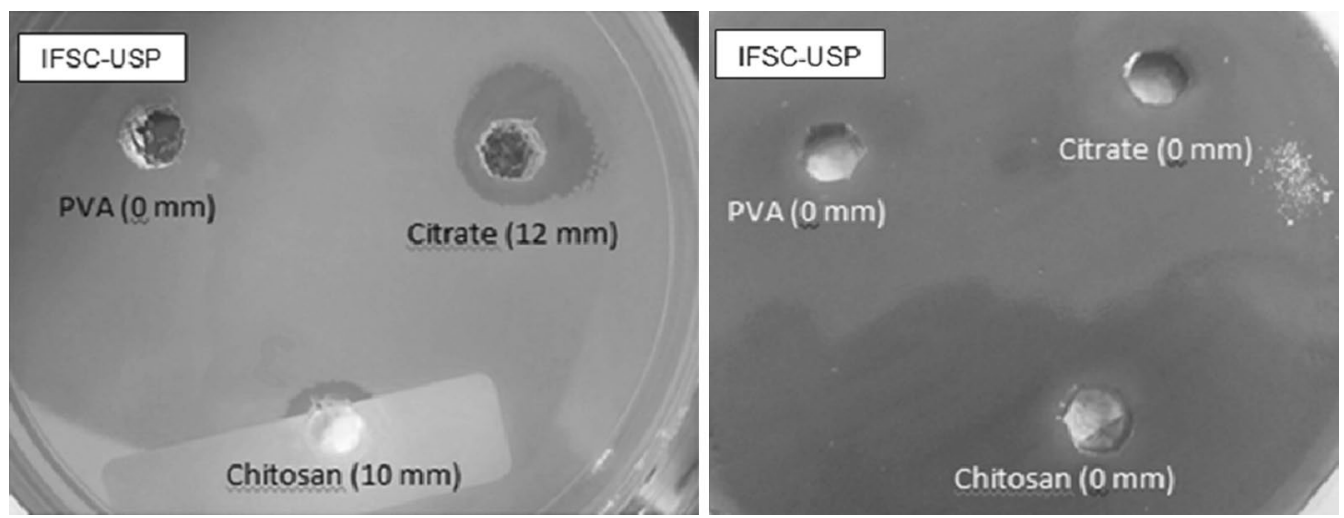

Fig. 4 Inhibition by diffusion in depth with AgNPs particles synthesized by IFSC-USP (citrate, chitosan and PVA AgNPs), for P. aeruginosa INCQS 230 in agar MHA (left) and the same microorganism in MHA blood $5 \%$ (right)

\section{Determination of minimum inhibitory concentration (MIC)} The analysis of $\mathrm{MIC}_{90}$ results were grouped in MDR and susceptible isolates (Table 2), the results against Grampositive and Gram-negative were similar for chitosan AgNPs $\left(6.75 \mu \mathrm{g} \mathrm{mL}^{-1}\right)$, PVA AgNPs $\left(\geq 54 \mu \mathrm{g} \mathrm{mL}^{-1}\right)$ and commercial AgNPs $\left(\geq 10 \mu \mathrm{g} \mathrm{mL}^{-1}\right)$. The $\mathrm{MIC}_{90}$ for citrate AgNPs was higher against susceptible Gram-positive isolates $\left(13.5 \mu \mathrm{g} \mathrm{mL}^{-1}\right)$ than against susceptible Gramnegative and MDR $\left(6.75 \mu \mathrm{g} \mathrm{mL}^{-1}\right)$. Regarding the silver controls, $\mathrm{Ag}$ sulfadiazine and $\mathrm{Ag}$ nitrate, the $\mathrm{MIC}_{90}$ were respectively $\geq 27$ and $13.5 \mu \mathrm{g} \mathrm{mL}^{-1}$, for Gram-positive isolates and 13.5 and $6.75 \mu \mathrm{g} \mathrm{mL}^{-1}$ for Gram-negative, independent of its antimicrobial resistance profile. In all tests the control silver sulfadiazine showed higher $\mathrm{MIC}_{90}$ results than citrate and chitosan AgNPs, while silver nitrate showed similar results to citrate AgNPs, except for a lower inhibitory effect against MDR Gram-positive $\left(13.5 \mu \mathrm{g} \mathrm{mL}^{-1}\right)$. It was not possible to define the exact $\mathrm{MIC}_{90}$ values for commercial AgNPs $\left(\geq 10 \mu \mathrm{g} \mathrm{mL}^{-1}\right)$ and PVA AgNPs, the less potent AgNPs particle evaluated was $\geq 54 \mu \mathrm{g} \mathrm{mL}^{-1}$ (Fig. 6). Summarizing, the effect of AgNPs was similar regardless of antimicrobial resistance profile and showed better activity against Gram-negative.

\section{Determination of minimum bactericidal concentration (MBC)}

Citrate AgNPs and chitosan AgNPs had higher bactericidal effect than PVA AgNPs and all controls. Both citrate and chitosan achieved bactericidal effect over $97 \%$ of antimicrobial-susceptible bacteria and 93 and $94 \%$ respectively for MDR. For controls, silver nitrate was bactericidal over $83 \%$ of both MDR and antimicrobialsusceptible bacteria while silver sulfadiazine was 61 and $67 \%$ respectively (Fig. 7). Citrate AgNPs were bacteriostatic against three $A$. baumannii MDR isolates, with $\mathrm{MBC} / \mathrm{MIC} \geq 8$ [26]. The results showed higher bactericidal effect against antimicrobial-susceptible bacteria than against MDR (Table 2).

\section{Time-kill}

The most significant reduction in the number of cfu in the shortest period of time occurred using chitosan AgNPs against the MSSA isolate (4 dilutions) and MRSA isolate (3 dilutions). After $12 \mathrm{~h}$ the MDR microorganism decreased its multiplication reaching the initial number of cfu, while the multiplication of antimicrobial-susceptible isolates remained until the end of $24 \mathrm{~h}$, the low counts was reached after the 6th hour (Fig. 8). Among Gramnegative the greatest reduction was observed for chitosan AgNPs against MDR-K. pneumoniae (4 dilutions) and against the antimicrobial-susceptible isolate of $E$. aerogenes (4 dilutions). Both remained at $10^{1} \mathrm{cfu} \mathrm{mL}^{-1}$ at the end of $24 \mathrm{~h}$ (Fig. 9).

As expected, the growth of the microorganisms tested in sub-inhibitory concentrations showed similar behavior when compared to positive controls, without AgNPs. The curves also showed the inhibitory effect of blood on the activity of all silver compounds and controls.

The present study evaluated the in vitro activity of three AgNPs stabilized with different compounds, against clinical isolates including MDR bacteria. The activity of AgNPs using diffusion in solid media and the MIC methods showed similar effect against MDR and antimicrobial-susceptible isolates, with a higher effect against Gram-negative isolates. The best inhibitory effect was achieved with citrate and chitosan AgNPs against Gram-negative bacteria.

The AgNPs showed a good in vitro activity against Gram-negative with different mechanism of resistance, including Enterobacteria harboring carbapenemase (KPC), Acinetobacter harboring oxacilinases, and P. aeruginosa harboring metallo-beta-lactamases. Interesting 

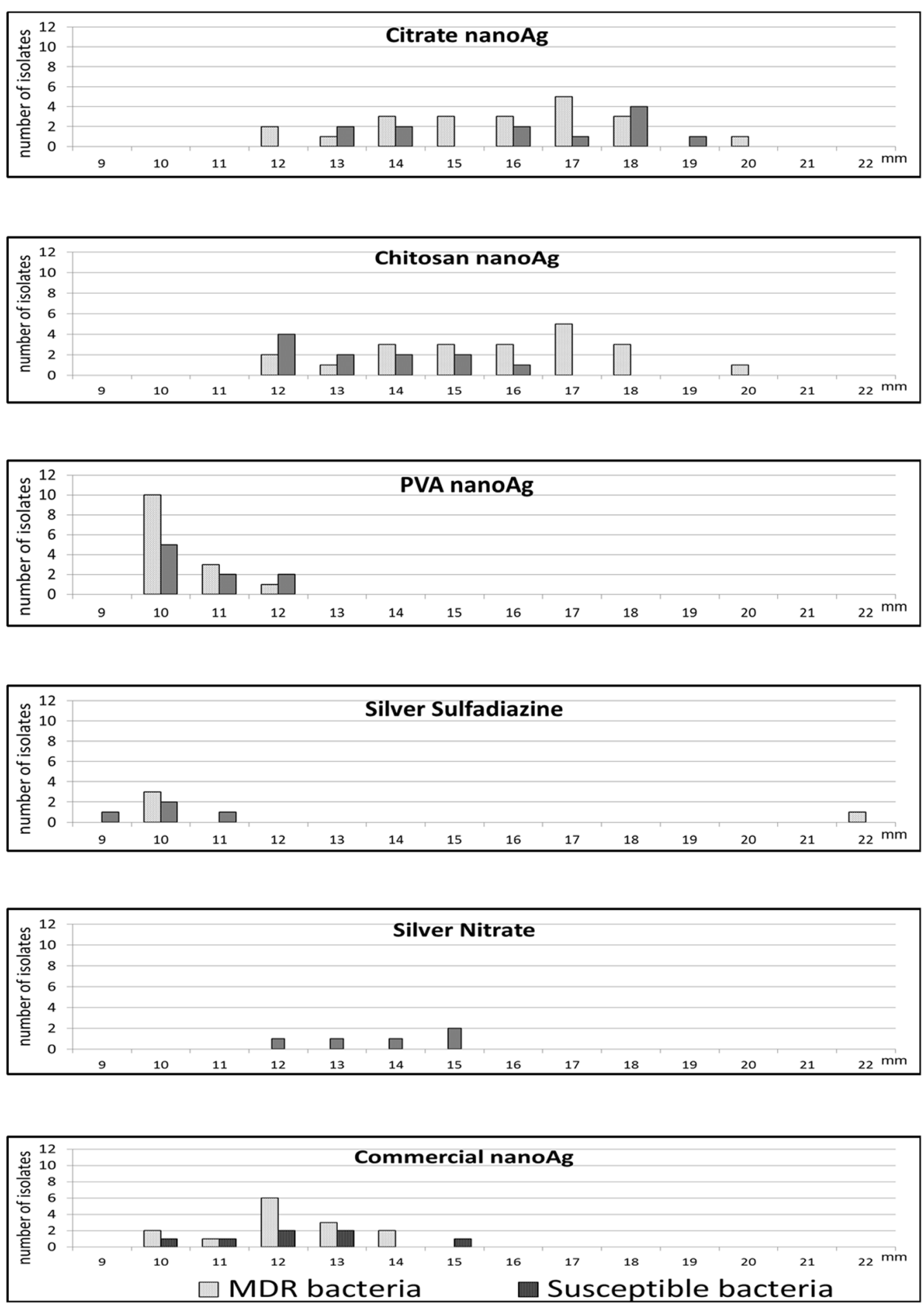

Fig. 5 Distribution of size of inhibition zones $(\mathrm{mm}$ ) obtained by diffusion in depth with AgNPs (citrate, chitosan and PVA) and controls against multidrug-resistant (MDR) $(n=54)$ and antimicrobial-susceptible bacteria $(n=36)$. Asterisk Only inhibition zones $>6$ mm were presented. All silver compounds showed absence of inhibition zones $(<6 \mathrm{~mm})$ when tested in MHA $5 \%$ blood against the same microorganisms 
Table 2 MIC, MIC ${ }_{50}, M C_{90}$ and MBC for MDR $(n=56)$ and antimicrobial susceptible microorganisms $(n=34)$

\begin{tabular}{|c|c|c|c|c|c|c|c|c|c|c|c|c|}
\hline \multirow[t]{2}{*}{ Microorganism } & \multicolumn{2}{|c|}{ Citrate } & \multicolumn{2}{|c|}{ Chitosan } & \multicolumn{2}{|l|}{ PVA } & \multicolumn{2}{|c|}{ Ag Sulfadiazin } & \multicolumn{2}{|c|}{ Ag Nitrate } & \multicolumn{2}{|c|}{$\begin{array}{l}\text { Commercial } \\
\text { AgNPs }\end{array}$} \\
\hline & MIC & MBC & MIC & MBC & MIC & MBC & MIC & MBC & MIC & MBC & MIC & MBC \\
\hline A. baumannii MDR (A1) & 3.4 & 13.5 & 6.7 & 6.7 & $\geq 54$ & $\geq 54$ & 6.7 & 6.7 & 3.4 & 3.4 & $\geq 10$ & $\geq 10$ \\
\hline A. baumannii MDR (A2) & 3.4 & 3.4 & 6.7 & 6.7 & 27 & 27 & 6.7 & 6.7 & 3.4 & 3.4 & $\geq 10$ & $\geq 10$ \\
\hline A. baumannii MDR (A3) & 3.4 & 13.5 & 6.7 & 6.7 & 13.5 & 13.5 & 6.7 & 6.7 & 3.4 & 3.4 & $\geq 10$ & $\geq 10$ \\
\hline A. baumannii MDR (A4) & 3.4 & 13.5 & 6.7 & 6.7 & $\geq 54$ & $\geq 54$ & 13.5 & 13.5 & 3.4 & 3.4 & $\geq 10$ & $\geq 10$ \\
\hline A. baumannii MDR (A5) & 3.4 & 13.5 & 6.7 & 6.7 & 13.5 & 13.5 & 6.7 & 6.7 & 3.4 & 3.4 & $\geq 10$ & $\geq 10$ \\
\hline A. baumannii MDR (A6) & 3.4 & 13.5 & 6.7 & 6.7 & 13.5 & 13.5 & 6.7 & 6.7 & 3.4 & 3.4 & $\geq 10$ & $\geq 10$ \\
\hline A. baumannii MDR (A7) & 3.4 & 27 & 6.7 & 6.7 & 13.5 & 27 & 6.7 & 13.5 & 3.4 & 13.5 & $\geq 10$ & $\geq 10$ \\
\hline A. baumannii MDR (A8) & 3.4 & 27 & 6.7 & 27 & 13.5 & 13.5 & 6.7 & 13.5 & 3.4 & 13.5 & $\geq 10$ & $\geq 10$ \\
\hline A. baumannii MDR (A9) & 3.4 & 27 & 6.7 & 6.7 & 13.5 & 13.5 & 6.7 & 6.7 & 3.4 & 3.4 & $\geq 10$ & $\geq 10$ \\
\hline A. baumannii MDR (A10) & 3.4 & 3.4 & 6.7 & 6.7 & 13.5 & 13.5 & 13.5 & 13.5 & 3.4 & 3.4 & $\geq 10$ & $\geq 10$ \\
\hline A. baumannii MDR (A11) & 3.4 & 13.5 & 6.7 & 6.7 & 13.5 & 27 & 6.7 & 6.7 & 3.4 & 3.4 & $\geq 10$ & $\geq 10$ \\
\hline A. baumannii MDR (A12) & 3.4 & 3.4 & 6.7 & 6.7 & 27 & 27 & 13.5 & 13.5 & 3.4 & 3.4 & $\geq 10$ & $\geq 10$ \\
\hline $\mathrm{MIC}_{50}$ A. baumannii MDR & 3.4 & - & 6.7 & - & 13.5 & - & 6.7 & - & 3.4 & - & $\geq 10$ & - \\
\hline $\mathrm{MIC}_{90}$ A. baumannii MDR & 3.4 & - & 6.7 & - & $\geq 54$ & - & 13.5 & - & 3.4 & - & $\geq 10$ & - \\
\hline A. baumannii S (AS1) & 3.4 & 3.4 & 3.4 & 6.7 & 6.7 & 13.5 & 6.7 & 6.7 & 3.4 & 3.4 & $\geq 10$ & $\geq 10$ \\
\hline A. baumannii S (AS2) & 1.6 & 3.4 & 1.6 & 1.6 & 6.7 & 13.5 & 6.7 & 13.5 & 3.4 & 3.4 & $\geq 10$ & $\geq 10$ \\
\hline A. baumannii S (AS3) & 3.4 & 3.4 & 3.4 & 3.4 & 6.7 & 13.5 & 6.7 & 6.7 & 3.4 & 3.4 & $\geq 10$ & $\geq 10$ \\
\hline A. baumannii S (AS4) & 3.4 & 3.4 & 3.4 & 3.4 & 27 & 27 & 6.7 & 6.7 & 3.4 & 3.4 & $\geq 10$ & $\geq 10$ \\
\hline A. baumannii S (AS5) & 3.4 & 3.4 & 3.4 & 6.7 & $\geq 54$ & $\geq 54$ & 6.7 & 6.7 & 3.4 & 3.4 & $\geq 10$ & $\geq 10$ \\
\hline $\mathrm{MIC}_{50}$ A. baumannii S & 3.4 & - & 3.4 & - & 6.7 & - & 6.7 & - & 3.4 & - & $\geq 10$ & - \\
\hline $\mathrm{MIC}_{90}$ A. baumannii S & 3.4 & - & 3.4 & - & $\geq 54$ & - & 6.7 & - & 3.4 & - & $\geq 10$ & - \\
\hline S. maltophilia MDR (MO9) & 1.6 & 13.5 & 6.7 & 6.7 & $\geq 54$ & $\geq 54$ & 6.7 & 6.7 & 3.4 & 3.4 & $\geq 10$ & $\geq 10$ \\
\hline S. maltophilia MDR (MO11) & 1.6 & 3.4 & 6.7 & 6.7 & $\geq 54$ & $\geq 54$ & 6.7 & 6.7 & 6.7 & 6.7 & $\geq 10$ & $\geq 10$ \\
\hline $\mathrm{MIC}_{50}$ S. maltophilia MDR & 1.6 & - & 6.7 & - & $\geq 54$ & - & 6.7 & - & 3.4 & - & $\geq 10$ & - \\
\hline $\mathrm{MIC}_{90}$ S. maltophilia MDR & 1.6 & - & 6.7 & - & $\geq 54$ & - & 6.7 & - & 6.7 & - & $\geq 10$ & - \\
\hline P. aeruginosa MDR (P1) & 3.4 & 3.4 & 6.7 & 13.5 & 27 & $\geq 54$ & 6.7 & 6.7 & 1.6 & 1.6 & $\geq 10$ & $\geq 10$ \\
\hline P. aeruginosa MDR (P2) & 3.4 & 6.7 & 6.7 & 13.5 & 13.5 & 27 & 13.5 & 13.5 & 3.4 & 6.7 & $\geq 10$ & $\geq 10$ \\
\hline P. aeruginosa MDR (P3) & 3.4 & 3.4 & 6.7 & 13.5 & 13.5 & 27 & 13.5 & 13.5 & 3.4 & 3.4 & $\geq 10$ & $\geq 10$ \\
\hline P. aeruginosa MDR (P4) & 3.4 & 13.5 & 6.7 & 13.5 & 13.5 & $\geq 54$ & 13.5 & 13.5 & 6.7 & $\geq 27$ & $\geq 10$ & $\geq 10$ \\
\hline P. aeruginosa MDR (P5) & 3.4 & 3.4 & 6.7 & 13.5 & 13.5 & 27 & 6.7 & 6.7 & 3.4 & 6.7 & $\geq 10$ & $\geq 10$ \\
\hline $\mathrm{MIC}_{50}$ P. aeruginosa MDR & 3.4 & - & 6.7 & - & 13.5 & - & 13.5 & - & 3.4 & - & $\geq 10$ & - \\
\hline $\mathrm{MIC}_{90}$ P. aeruginosa MDR & 3.4 & - & 6.7 & - & 27 & - & 13.5 & - & 6.7 & - & $\geq 10$ & - \\
\hline P. aeruginosa S (PS1) & 3.4 & 34 & 6.7 & 13.5 & 13.5 & 13.5 & 6.7 & 6.7 & 3.4 & 13.5 & $\geq 10$ & $\geq 10$ \\
\hline P. aeruginosa S (PS2) & 3.4 & 3.4 & 6.7 & 6.7 & 13.5 & $\geq 54$ & 13.5 & 13.5 & 6.7 & 13.5 & $\geq 10$ & $\geq 10$ \\
\hline P. aeruginosa S (PS3) & 3.4 & 1.6 & 3.4 & 3.4 & $\geq 54$ & $\geq 54$ & 13.5 & 13.5 & 6.7 & 6.7 & $\geq 10$ & $\geq 10$ \\
\hline P. aeruginosa S (PS4) & 1.6 & 1.6 & 3.4 & 6.7 & 13.5 & 13.5 & 3.4 & 13.5 & 1.6 & 3.4 & $\geq 10$ & $\geq 10$ \\
\hline P. aeruginosa S (PS5) & 1.6 & 3.4 & 6.7 & 13.5 & 13.5 & 27 & 6.7 & 6.7 & 3.4 & 3.4 & $\geq 10$ & $\geq 10$ \\
\hline P. aeruginosa S (PS6) & 1.6 & 3.4 & 6.7 & 13.5 & 6.7 & $\geq 54$ & 6.7 & 13.5 & 3.4 & 3.4 & $\geq 10$ & $\geq 10$ \\
\hline P. aeruginosa S (PS7) & 1.6 & 3.4 & 3.4 & 13.5 & 6.7 & 13.5 & 13.5 & 13.5 & 6.7 & 6.7 & $\geq 10$ & $\geq 10$ \\
\hline P. aeruginosa ATCC 27853 S (MO18) & 3.4 & 6.7 & 13.5 & 13.5 & 13.5 & $\geq 54$ & 6.7 & 6.7 & 3.4 & 3.4 & $\geq 10$ & $\geq 10$ \\
\hline $\mathrm{MIC}_{50}$ P. aeruginosaS & 1.6 & - & 6.7 & - & 13.5 & - & 6.7 & - & 3.4 & - & $\geq 10$ & - \\
\hline $\mathrm{MIC}_{90}$ P. aeruginosa S & 3.4 & - & 13.5 & - & $\geq 54$ & - & 13.5 & - & 6.7 & - & $\geq 10$ & - \\
\hline Enterobacteriaceae MDR (K1) & 6.7 & 6.7 & 6.7 & 6.7 & $\geq 54$ & $\geq 54$ & 13.5 & 13.5 & 6.7 & $\geq 27$ & $\geq 10$ & $\geq 10$ \\
\hline Enterobacteriaceae MDR (K2) & 6.7 & 6.7 & 6.7 & 6.7 & $\geq 54$ & $\geq 54$ & 13.5 & 13.5 & 6.7 & 6.7 & $\geq 10$ & $\geq 10$ \\
\hline Enterobacteriaceae MDR (K3) & 6.7 & 13.5 & 6.7 & 6.7 & 13.5 & $\geq 54$ & 13.5 & 13.5 & 6.7 & 6.7 & $\geq 10$ & $\geq 10$ \\
\hline Enterobacteriaceae MDR (K4) & 6.7 & 6.7 & 6.7 & 6.7 & $\geq 54$ & $\geq 54$ & 13.5 & 13.5 & 6.7 & 6.7 & $\geq 10$ & $\geq 10$ \\
\hline Enterobacteriaceae MDR (K5) & 6.7 & 6.7 & 6.7 & 6.7 & 27 & $\geq 54$ & 13.5 & 13.5 & 6.7 & 6.7 & $\geq 10$ & $\geq 10$ \\
\hline
\end{tabular}


Table 2 continued

\begin{tabular}{|c|c|c|c|c|c|c|c|c|c|c|c|c|}
\hline \multirow[t]{2}{*}{ Microorganism } & \multicolumn{2}{|c|}{ Citrate } & \multicolumn{2}{|c|}{ Chitosan } & \multicolumn{2}{|l|}{ PVA } & \multicolumn{2}{|c|}{ Ag Sulfadiazin } & \multicolumn{2}{|c|}{ Ag Nitrate } & \multicolumn{2}{|c|}{$\begin{array}{l}\text { Commercial } \\
\text { AgNPs }\end{array}$} \\
\hline & MIC & MBC & MIC & MBC & MIC & MBC & MIC & MBC & MIC & MBC & MIC & MBC \\
\hline Enterobacteriaceae MDR (K6) & 6.7 & 13.5 & 6.7 & 6.7 & 27 & $\geq 54$ & 13.5 & 13.5 & 6.7 & 6.7 & $\geq 10$ & $\geq 10$ \\
\hline Enterobacteriaceae MDR (K7) & 6.7 & 6.7 & 6.7 & 6.7 & 27 & 27 & 13.5 & 13.5 & 6.7 & 6.7 & $\geq 10$ & $\geq 10$ \\
\hline Enterobacteriaceae MDR (K9) & 6.7 & 6.7 & 6.7 & 6.7 & $\geq 54$ & $\geq 54$ & 13.5 & 13.5 & 6.7 & 6.7 & $\geq 10$ & $\geq 10$ \\
\hline Enterobacteriaceae MDR (K14) & 6.7 & 27 & 6.7 & 13.5 & $\geq 54$ & $\geq 54$ & 13.5 & 13.5 & 6.7 & $\geq 27$ & $\geq 10$ & $\geq 10$ \\
\hline Enterobacteriaceae MDR (MO13) & 3.3 & 3.3 & 3.3 & 3.3 & 13.5 & 13.5 & 13.5 & 13.5 & 6.7 & $\geq 27$ & $\geq 10$ & $\geq 10$ \\
\hline Enterobacteriaceae MDR (MO8) & 3.3 & 13.5 & 6.7 & 6.7 & $\geq 54$ & $\geq 54$ & 13.5 & 13.5 & 3.4 & 3.4 & $\geq 10$ & $\geq 10$ \\
\hline Enterobacteriaceae MDR (MO10) & 3.3 & 3.3 & 6.7 & 6.7 & $\geq 54$ & $\geq 54$ & 13.5 & 13.5 & 6.7 & 6.7 & $\geq 10$ & $\geq 10$ \\
\hline $\mathrm{MIC}_{50}$ Enterobacteriaceae MDR & 6.7 & - & 6.7 & - & $\geq 54$ & - & 13.5 & - & 6.7 & - & $\geq 10$ & - \\
\hline $\mathrm{MIC}_{90}$ Enterobacteriaceae MDR & 6.7 & - & 6.7 & - & $\geq 54$ & - & 13.5 & - & 6.7 & - & $\geq 10$ & - \\
\hline Enterobacteriaceae S (ENB1) & 3.4 & 3.4 & 6.7 & 6.7 & 27 & 27 & 13.5 & 13.5 & 6.7 & 6.7 & $\geq 10$ & $\geq 10$ \\
\hline Enterobacteriaceae S (ENB2) & 6.7 & 6.7 & 3.4 & 6.7 & $\geq 54$ & $\geq 54$ & 13.5 & 13.5 & 13.5 & 13.5 & $\geq 10$ & $\geq 10$ \\
\hline Enterobacteriaceae S (ENB3) & 3.4 & 3.4 & 3.4 & 3.4 & 13.5 & 13.5 & 13.5 & 13.5 & 3.4 & 6.7 & $\geq 10$ & $\geq 10$ \\
\hline Enterobacteriaceae S (ENB4) & 6.7 & 6.7 & 3.4 & 6.7 & 27 & 27 & 13.5 & 13.5 & 6.7 & $\geq 27$ & $\geq 10$ & $\geq 10$ \\
\hline Enterobacteriaceae S (ENB5) & 6.7 & 6.7 & 6.7 & 6.7 & 27 & 27 & 13.5 & $\geq 27$ & 6.7 & 6.7 & $\geq 10$ & $\geq 10$ \\
\hline Enterobacteriaceae S (ENB6) & 6.7 & 6.7 & 6.7 & 6.7 & 27 & 27 & 13.5 & 13.5 & 6.7 & 6.7 & $\geq 10$ & $\geq 10$ \\
\hline Enterobacteriaceae S (ENB7) & 6.7 & 6.7 & 3.4 & 3.4 & 13.5 & 13.5 & 13.5 & 13.5 & 6.7 & 6.7 & $\geq 10$ & $\geq 10$ \\
\hline Enterobacteriaceae S (ENB8) & 3.4 & 3.4 & 3.4 & 3.4 & 13.5 & 27 & 13.5 & 13.5 & 6.7 & 6.7 & $\geq 10$ & $\geq 10$ \\
\hline Enterobacteriaceae S (ENB9) & 3.4 & 27 & 3.4 & 3.4 & $\geq 54$ & $\geq 54$ & 13.5 & 13.5 & 6.7 & 6.7 & $\geq 10$ & $\geq 10$ \\
\hline $\mathrm{MIC}_{50}$ Enterobacteriaceae S & 6.7 & - & 3.4 & - & 27 & - & 13.5 & - & 6.7 & - & $\geq 10$ & - \\
\hline $\mathrm{MIC}_{90}$ Enterobacteriaceae $\mathrm{S}$ & 6.7 & - & 6.7 & - & $\geq 54$ & - & 13.5 & - & 13.5 & - & $\geq 10$ & - \\
\hline S. aureus MRSA (S1) & 6.7 & 6.7 & 3.4 & 27 & $\geq 54$ & $\geq 54$ & 13.5 & 13.5 & 13.5 & $\geq 27$ & $\geq 10$ & $\geq 10$ \\
\hline S. aureus MRSA (S2) & 6.7 & 13.5 & 3.4 & 6.7 & $\geq 54$ & $\geq 54$ & $\geq 27$ & $\geq 27$ & 13.5 & $\geq 27$ & $\geq 10$ & $\geq 10$ \\
\hline S. aureus MRSA (S3) & 6.7 & 13.5 & 3.4 & 3.4 & $\geq 54$ & $\geq 54$ & $\geq 27$ & $\geq 27$ & 13.5 & $\geq 27$ & $\geq 10$ & $\geq 10$ \\
\hline S. aureus MRSA (S4) & 6.7 & 13.5 & 3.4 & 3.4 & $\geq 54$ & $\geq 54$ & $\geq 27$ & $\geq 27$ & 13.5 & $\geq 27$ & $\geq 10$ & $\geq 10$ \\
\hline S. aureus MRSA (S5) & 6.7 & 13.5 & 3.4 & 3.4 & $\geq 54$ & $\geq 54$ & 13.5 & 13.5 & 13.5 & 13.5 & $\geq 10$ & $\geq 10$ \\
\hline S. aureus MRSA (S6) & 6.7 & 13.5 & 3.4 & 27 & $\geq 54$ & $\geq 54$ & $\geq 27$ & $\geq 27$ & 13.5 & 13.5 & $\geq 10$ & $\geq 10$ \\
\hline S. aureus MRSA (S7) & 6.7 & 13.5 & 3.4 & 3.4 & $\geq 54$ & $\geq 54$ & $\geq 27$ & $\geq 27$ & 13.5 & 13.5 & $\geq 10$ & $\geq 10$ \\
\hline S. aureus MRSA (S8) & 6.7 & 13.5 & 3.4 & 3.4 & 27 & 27 & $\geq 27$ & $\geq 27$ & 13.5 & 13.5 & $\geq 10$ & $\geq 10$ \\
\hline S. aureus MRSA (MO1) & 6.7 & 27 & 3.4 & 27 & $\geq 54$ & $\geq 54$ & $\geq 27$ & $\geq 27$ & 13.5 & 13.5 & $\geq 10$ & $\geq 10$ \\
\hline S. aureus MRSA (MO2) & 6.7 & 13.5 & 3.4 & 3.4 & $\geq 54$ & $\geq 54$ & 13.5 & $\geq 27$ & 13.5 & 13.5 & $\geq 10$ & $\geq 10$ \\
\hline S. aureus MRSA (MO3) & 6.7 & 13.5 & 6.7 & 6.7 & $\geq 54$ & $\geq 54$ & $\geq 27$ & $\geq 27$ & 13.5 & 13.5 & $\geq 10$ & $\geq 10$ \\
\hline S. aureus MRSA (MO4) & 6.7 & 13.5 & 6.7 & 27 & $\geq 54$ & $\geq 54$ & $\geq 27$ & $\geq 27$ & 13.5 & $\geq 27$ & $\geq 10$ & $\geq 10$ \\
\hline S. aureus MRSA (MO5) & 6.7 & 13.5 & 6.7 & 27 & $\geq 54$ & $\geq 54$ & $\geq 27$ & $\geq 27$ & 13.5 & 13.5 & $\geq 10$ & $\geq 10$ \\
\hline S. aureus MRSA (MO6) & 13.5 & 27 & 6.7 & 6.7 & $\geq 54$ & $\geq 54$ & $\geq 27$ & $\geq 27$ & 13.5 & 13.5 & $\geq 10$ & $\geq 10$ \\
\hline S. aureus MRSA (MO7) & 13.5 & 13.5 & 6.7 & 6.7 & $\geq 54$ & $\geq 54$ & $\geq 27$ & $\geq 27$ & 13.5 & 13.5 & $\geq 10$ & $\geq 10$ \\
\hline $\mathrm{MIC}_{50}$ S. aureus MRSA & 6.7 & - & 3.4 & - & $\geq 54$ & - & $\geq 27$ & - & 13.5 & - & $\geq 10$ & - \\
\hline $\mathrm{MIC}_{90}$ S. aureus MRSA & 13.5 & - & 6.7 & - & $\geq 54$ & - & $\geq 27$ & - & 13.5 & - & $\geq 10$ & - \\
\hline S. aureus MSSA (MSSA1) & 13.5 & 27 & 6.7 & 27 & $\geq 54$ & $\geq 54$ & $\geq 27$ & $\geq 27$ & 13.5 & 13.5 & $\geq 10$ & $\geq 10$ \\
\hline S. aureus MSSA (MSSA2) & 6.7 & 13.5 & 6.7 & 6.7 & $\geq 54$ & $\geq 54$ & $\geq 27$ & $\geq 27$ & 13.5 & 13.5 & $\geq 10$ & $\geq 10$ \\
\hline S. aureus MSSA (MSSA3) & 6.7 & 13.5 & 6.7 & 6.7 & $\geq 54$ & $\geq 54$ & $\geq 27$ & $\geq 27$ & 13.5 & 13.5 & $\geq 10$ & $\geq 10$ \\
\hline S. aureus MSSA (MSSA4) & 6.7 & 13.5 & 6.7 & $\geq 54$ & $\geq 54$ & $\geq 54$ & $\geq 27$ & $\geq 27$ & 13.5 & 13.5 & $\geq 10$ & $\geq 10$ \\
\hline S. aureus MSSA (MSSA5) & 6.7 & 13.5 & 6.7 & 6.7 & $\geq 54$ & $\geq 54$ & $\geq 27$ & $\geq 27$ & 13.5 & 13.5 & $\geq 10$ & $\geq 10$ \\
\hline S. aureus MSSA (MSSA6) & 6.7 & 13.5 & 6.7 & 6.7 & $\geq 54$ & $\geq 54$ & $\geq 27$ & $\geq 27$ & 13.5 & 13.5 & $\geq 10$ & $\geq 10$ \\
\hline S. aureus ATCC29213 (MO17) & 13.5 & 27 & 3.4 & 6.7 & $\geq 54$ & $\geq 54$ & $\geq 27$ & $\geq 27$ & 13.5 & $\geq 27$ & $\geq 10$ & $\geq 10$ \\
\hline S. epidermidis INCQS198 (MO16) & 6.7 & 6.7 & 6.7 & 6.7 & 27 & 27 & 6.7 & 13.5 & 6.7 & 6.7 & $\geq 10$ & $\geq 10$ \\
\hline $\mathrm{MIC}_{50}$ S. aureus MSSA & 6.7 & - & 6.7 & - & $\geq 54$ & - & $\geq 27$ & - & 13.5 & - & $\geq 10$ & - \\
\hline $\mathrm{MIC}_{90}$ S. aureus MSSA & 13.5 & - & 6.7 & - & $\geq 54$ & - & $\geq 27$ & - & 13.5 & - & $\geq 10$ & - \\
\hline Enterococcus sp. VRE (V1) & 6.7 & 27 & 6.7 & 27 & $\geq 54$ & $\geq 54$ & $\geq 27$ & $\geq 27$ & 13.5 & 13.5 & $\geq 10$ & $\geq 10$ \\
\hline
\end{tabular}


Table 2 continued

\begin{tabular}{|c|c|c|c|c|c|c|c|c|c|c|c|c|}
\hline \multirow[t]{2}{*}{ Microorganism } & \multicolumn{2}{|c|}{ Citrate } & \multicolumn{2}{|c|}{ Chitosan } & \multicolumn{2}{|l|}{ PVA } & \multicolumn{2}{|c|}{ Ag Sulfadiazin } & \multicolumn{2}{|c|}{ Ag Nitrate } & \multicolumn{2}{|c|}{$\begin{array}{l}\text { Commercial } \\
\text { AgNPs }\end{array}$} \\
\hline & MIC & MBC & MIC & MBC & MIC & MBC & MIC & $\mathrm{MBC}$ & MIC & MBC & MIC & MBC \\
\hline Enterococcus sp. VRE (V2) & 6.7 & 27 & 6.7 & 6.7 & $\geq 54$ & $\geq 54$ & $\geq 27$ & $\geq 27$ & 13.5 & 13.5 & $\geq 10$ & $\geq 10$ \\
\hline Enterococcus sp. VRE (V3) & 6.7 & 27 & 6.7 & 6.7 & $\geq 54$ & $\geq 54$ & $\geq 27$ & $\geq 27$ & 13.5 & 13.5 & $\geq 10$ & $\geq 10$ \\
\hline Enterococcus sp. VRE (V4) & 6.7 & 27 & 6.7 & 13.5 & $\geq 54$ & $\geq 54$ & $\geq 27$ & $\geq 27$ & 13.5 & 13.5 & $\geq 10$ & $\geq 10$ \\
\hline Enterococcus sp. VRE (V5) & 6.7 & 27 & 6.7 & 13.5 & 27 & $\geq 54$ & $\geq 27$ & $\geq 27$ & 13.5 & 13.5 & $\geq 10$ & $\geq 10$ \\
\hline Enterococcus sp. VRE (V6) & 6.7 & 13.5 & 3.4 & 13.5 & $\geq 54$ & $\geq 54$ & $\geq 27$ & $\geq 27$ & 6.7 & 13.5 & $\geq 10$ & $\geq 10$ \\
\hline Enterococcus sp. VRE (V7) & 6.7 & 27 & 3.4 & 6.7 & 27 & $\geq 54$ & $\geq 27$ & $\geq 27$ & 6.7 & 13.5 & $\geq 10$ & $\geq 10$ \\
\hline Enterococcus sp. VRE (V8) & 6.7 & 27 & 6.7 & 6.7 & 13.5 & 13.5 & $\geq 27$ & $\geq 27$ & 13.5 & 13.5 & $\geq 10$ & $\geq 10$ \\
\hline $\mathrm{MIC}_{50}$ Enterococcus sp. VRE & 6.7 & - & 6.7 & - & $\geq 54$ & - & $\geq 27$ & - & 13.5 & - & $\geq 10$ & - \\
\hline $\mathrm{MIC}_{90}$ Enterococcus sp. VRE & 6.7 & - & 6.7 & - & $\geq 54$ & - & $\geq 27$ & - & 13.5 & - & $\geq 10$ & - \\
\hline Enterococcus sp. VSE (VSE1) & 6.7 & 27 & 6.7 & 13.5 & 27 & $\geq 54$ & $\geq 27$ & $\geq 27$ & $\geq 27$ & $\geq 27$ & $\geq 10$ & $\geq 10$ \\
\hline Enterococcus sp. VSE (VSE2) & 6.7 & 6.7 & 6.7 & 13.5 & $\geq 54$ & $\geq 54$ & 13.5 & $\geq 27$ & $\geq 27$ & $\geq 27$ & $\geq 10$ & $\geq 10$ \\
\hline Enterococcus sp. VSE (VSE3) & 13.5 & 13.5 & 3.4 & 3.4 & $\geq 54$ & $\geq 54$ & $\geq 27$ & $\geq 27$ & $\geq 27$ & $\geq 27$ & $\geq 10$ & $\geq 10$ \\
\hline Enterococcus sp. VSE (VSE4) & 6.7 & 6.7 & 6.7 & 6.7 & $\geq 54$ & $\geq 54$ & 13.5 & 13.5 & 13.5 & 13.5 & $\geq 10$ & $\geq 10$ \\
\hline Enterococcus sp. VSE (VSE5) & 6.7 & 6.7 & 6.7 & 6.7 & $\geq 54$ & $\geq 54$ & 13.5 & 13.5 & 13.5 & 13.5 & $\geq 10$ & $\geq 10$ \\
\hline Enterococcus sp. VSE (VSE6) & 6.7 & 6.7 & 6.7 & 6.7 & $\geq 54$ & $\geq 54$ & 13.5 & $\geq 27$ & 13.5 & $\geq 27$ & $\geq 10$ & $\geq 10$ \\
\hline $\mathrm{MIC}_{50}$ Enterococcus sp. VSE & 6.7 & - & 6.7 & - & $\geq 54$ & - & 13.5 & - & 13.5 & - & $\geq 10$ & - \\
\hline $\mathrm{MIC}_{90}$ Enterococcus sp. VSE & 13.5 & - & 6.7 & - & $\geq 54$ & - & $\geq 27$ & - & $\geq 27$ & - & $\geq 10$ & - \\
\hline \multicolumn{13}{|l|}{$\operatorname{MDR}(N=54)$} \\
\hline $\mathrm{MIC}_{50} \mathrm{MDR}$ & 6.7 & - & 6.7 & - & $\geq 54$ & - & 13.5 & - & 6.7 & - & $\geq 10$ & - \\
\hline $\mathrm{MIC}_{90} \mathrm{MDR}$ & 6.7 & - & 6.7 & - & $\geq 54$ & - & $\geq 27$ & - & 13.5 & - & $\geq 10$ & - \\
\hline \multicolumn{13}{|l|}{ Susceptible $(\mathrm{N}=36)$} \\
\hline $\mathrm{MIC}_{50}$ Susceptible & 6.7 & - & 6.7 & - & $\geq 54$ & - & 13.5 & - & 6.7 & - & $\geq 10$ & - \\
\hline MIC $_{90}$ Susceptible & 6.7 & - & 6.7 & - & $\geq 54$ & - & $\geq 27$ & - & 13.5 & - & $\geq 10$ & - \\
\hline \multicolumn{13}{|l|}{ TOTAL $(\mathrm{N}=90)$} \\
\hline $\mathrm{MIC}_{50}$ & 6.7 & - & 6.7 & - & $\geq 54$ & - & 13.5 & - & 6.7 & - & $\geq 10$ & - \\
\hline $\mathrm{MIC}_{90}$ & 6.7 & - & 6.7 & - & $\geq 54$ & - & $\geq 27$ & - & 13.5 & - & $\geq 10$ & - \\
\hline
\end{tabular}

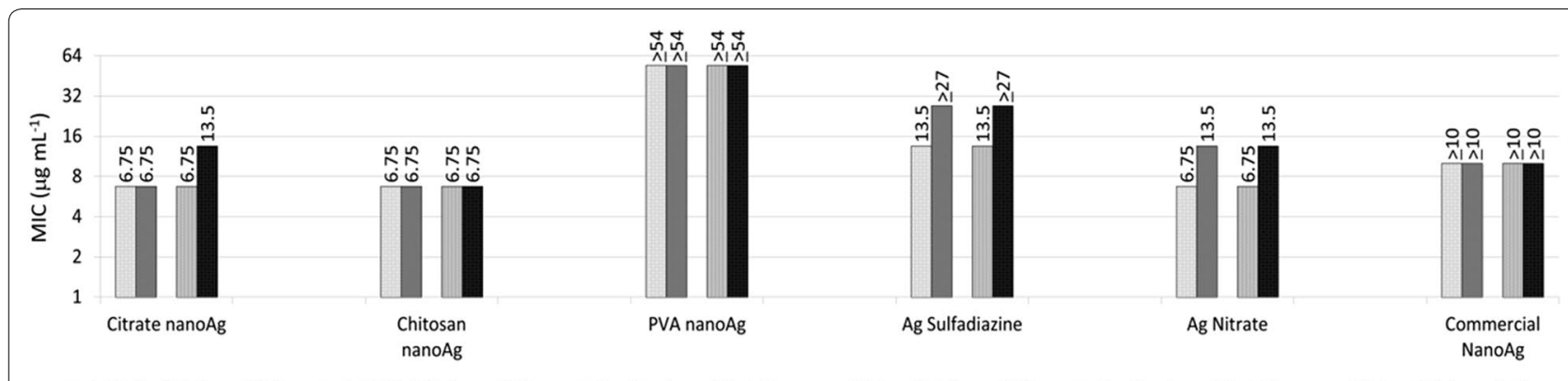

$\square$ MDR GN (n= 31) $\square$ MDR GP ( $n=23) \quad \square$ Antimicrobial-Susceptible GN ( $n=22) \quad$ Antimicrobial-Susceptible GP ( $n=14)$

Fig. $6 \mathrm{MIC}_{90}$ for AgNPs (citrate, chitosan and PVA) and controls comparing the subgroups MDR Gram-negative GN ( $n=31$ ) versus MDR Grampositive GP $(n=23)$ (totalizing $n=54)$ and antimicrobial-susceptible $G N(n=22)$ versus $G P(n=14)$ (totalizing $n=36)$

points of this study were the inclusion of MDR clinical isolates with previously studied mechanisms of resistance, paired with susceptible isolates, and the large number of isolates evaluated.

In an attempt to reduce the selection of resistant microorganisms, antimicrobials should have bactericidal effect which can be determined by the ratio MBC/MIC $\leq 4$ [26]. The present study showed the predominance of the bactericidal effect of AgNPs particles studied, similar to previously described [3]. The best bactericidal effect was achieved with citrate and chitosan AgNPs against susceptible bacteria. Citrate AgNPs showed the highest 


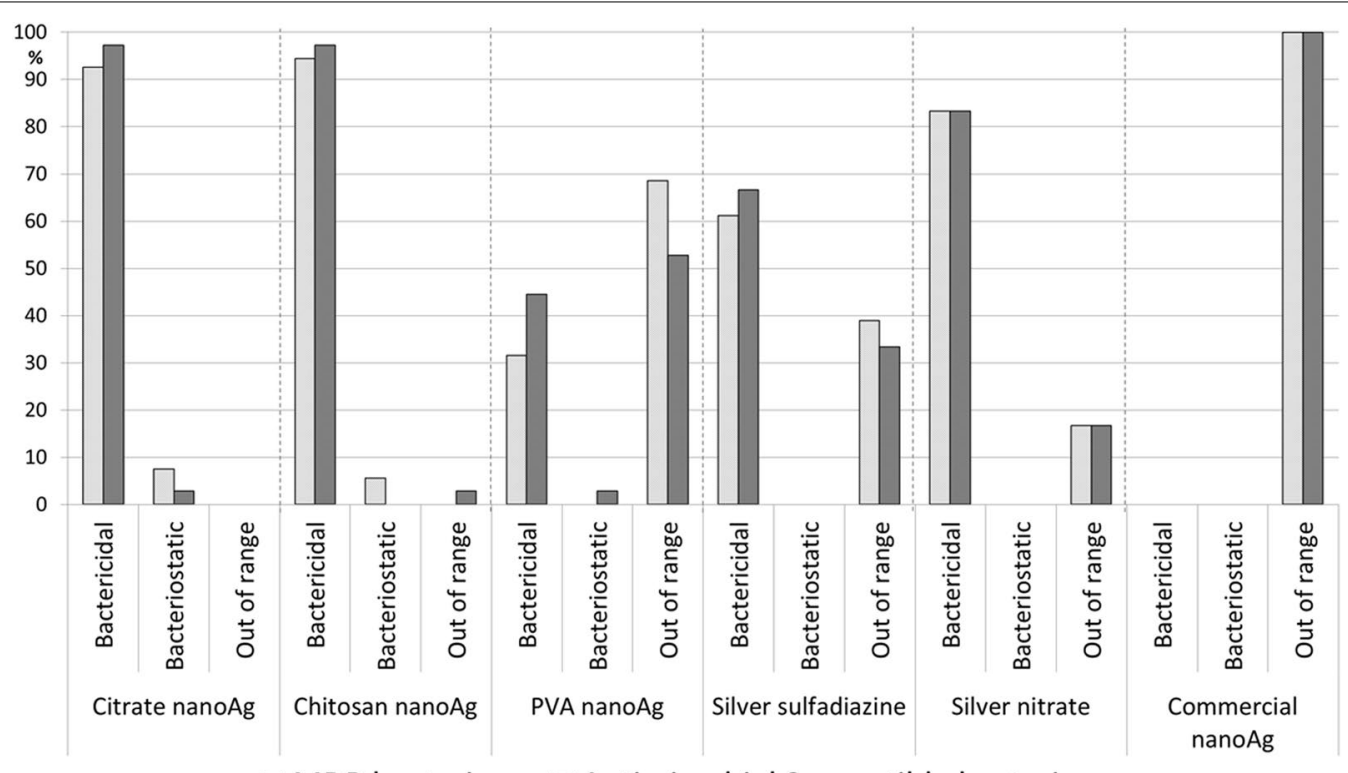

$\square$ MDR bacteria $\square$ Antimicrobial Susceptible bacteria

Fig. 7 Results of minimum bactericidal concentration/minimum inhibitory concentration (MBC/MIC) ratio of AgNPs particles (citrate, chitosan and PVA) and controls against multidrug-resistant (MDR) $(n=54)$ and susceptible $(n=36)$ bacteria

inhibitory effect against all isolates, but even better over Gram-negative. Chitosan AgNPs showed identical effect against Gram-positive and Gram-negative, independently of its mechanism of resistance. In agreement with the MIC results, the PVA AgNPs had the time kill curve with less inhibition for all isolates evaluated. The most significant reduction in the number of $\mathrm{cfu}$ in the shortest period of time was achieved with chitosan AgNPs against MRSA and K. pneumoniae harboring KPC.

The ISO Technical Committee on Nanotechnologies (TC 229) has recently published two new standards methods for analysis of antimicrobial effect [8] and toxicity of AgNPs [9]. However, a variety of other methodologies has been used in literature, as described by Jena et al. using colony-forming unit assay [27], Shrivastava et al. used methods of agar dilution [28] and Patil et al. used qualitative method of agar well diffusion to evaluate MIC of AgNPs [29]. This wide variety of methods makes the comparison of results a difficult task.

In the present study, diffusion in solid media, MIC, $\mathrm{MBC}$, and time-kill methods were evaluated using culture media with and without blood. In all tests some degree of interference of the blood on enriched media was detected, with a reduction of all silver (AgNPs, silver sulfadiazine, and silver nitrate) activity against the microorganisms tested. In the presence of blood, we observed smaller inhibition zones, higher MIC and MBC, and a reduction on the inhibitory effect of death curve with reduction. Thus, media supplement with blood should be avoided to evaluate in vitro activity of AgNPs.

The diffusion method in depth by AWD was previously used as screening test to detect in vitro activity of AgNPs against bacteria [11, 13]. It is an easy method to be use in routine microbiology laboratory. The inhibition zones obtained in the present study showed similar AgNPs activity against MDR and susceptible isolates and that citrate and chitosan AgNPs presented greater effect against the isolates similar with the results using micro dilution method. The $\mathrm{MIC}_{90}$ results reinforced and validated the diffusion method results. This results can be explained by the fact that AgNPs interaction with three vital components of cells: peptidoglycan cell wall, cytoplasmic membrane and biomolecules like ribosomal DNA and phosphorus and sulfur groups present in proteins $[7,30]$.

AgNP shows greater capacity and higher surface areato-volume ratio compared to silver salts [5, 30-32]. It is an aggregate of silver atoms up to $100 \mathrm{~nm}$ in diameter and its features change compared to both the ion and the bulk material in their chemical, mechanical, electrical, optical properties, catalytic activity, conductivity and biological effect. Silver is the more effective antimicrobial agent against bacteria, viruses and other eukaryotic microorganisms $[6,33]$ than other metals such as copper, titanium, magnesium, zinc or gold.

The impact of the nanosize on silver activity against bacteria was already well demonstrated, Morones et al. 


\section{Oxacillin-resistant $S$. aureus}

\section{Citrate $(\mathrm{MIC}=3.3 ; \mathrm{MBC}=27)$}

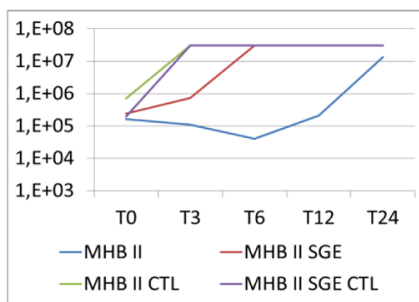

AgSulfad (MIC=13.5; MBC $\geq 27)$

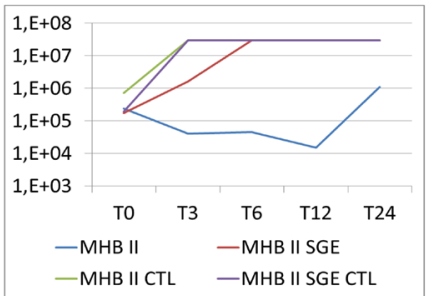

AgNitrate $(\mathrm{MIC}=13.5 ; \mathrm{MBC}=13.5)$

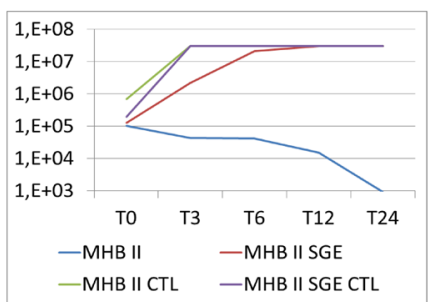

PVA $(M I C=27 ; M B C \geq 54)$

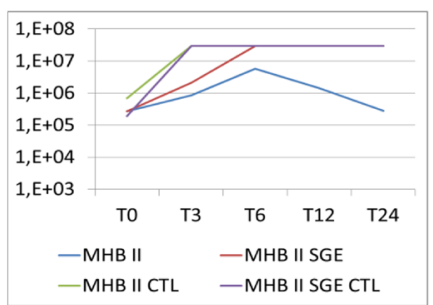

\section{Commercial $(M I C \geq 10 ; M B C \geq 10)$}

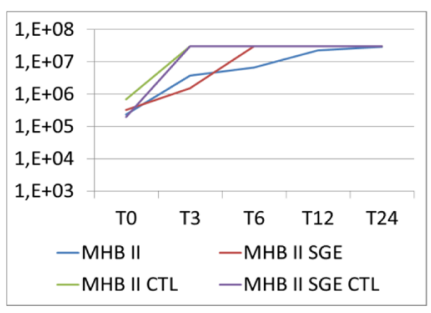

\section{Oxacillin-susceptible S. aureus}

\section{Citrate $(\mathrm{MIC}=6.7: \mathrm{MBC}=13.5)$}

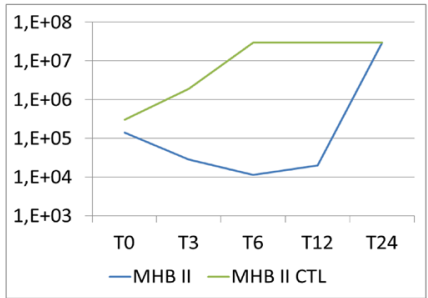

Chitosan $(\mathrm{MIC}=6.7 ; \mathrm{MBC}=6.7)$

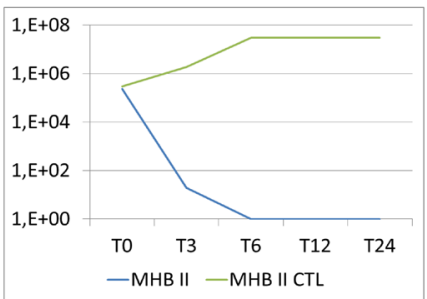

PVA $(M I C \geq 54 ; M B C \geq 54)$

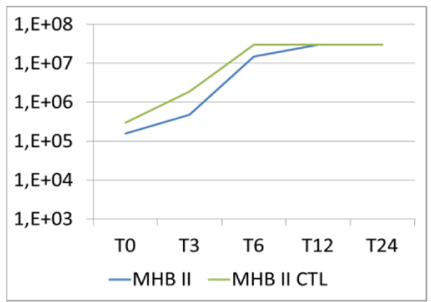

Commercial( $M I C \geq 10 ; M B C \geq 10)$

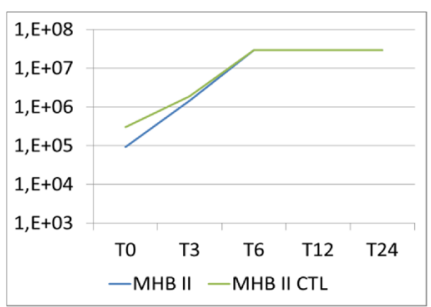

Fig. 8 Comparison of time-kill curves for one oxacillin-resistant S. aureus (MRSA) and one oxacillin-suscpetible S. aureus (MSSA) isolate, using AgNPs particles (citrate, chitosan and PVA) and controls (silver sulfadiazine, silver nitrate and commercial AgNPs). For MRSA was made the comparison using MHB II broth and MHB II blood $1.25 \%$. MHB II- Mueller-Hinton Broth cation adjusted and microorganism; MHB II CTL-control without microorganisms. MHB II SGE-microorganism and broth enriched with blood; MHB II CTL SGE-only broth and blood. For oxacilin-susceptible S. aureus (MSSA3), silver sulfadiazine and silver nitrate curves were not done due to high MICs (Ag Sulfad and Ag Nitrate: MIC $\geq 27 \mu \mathrm{g} \mathrm{mL}{ }^{-1} ; \mathrm{MBC}$ $\geq 27 \mu \mathrm{g} \mathrm{mL}^{-1}$ ) 


\section{Carbapenem-resistant K. pneumonia (KPC)}

Citrate $(\mathrm{MIC}=6.7 ; \mathrm{MBC}=13.5)$

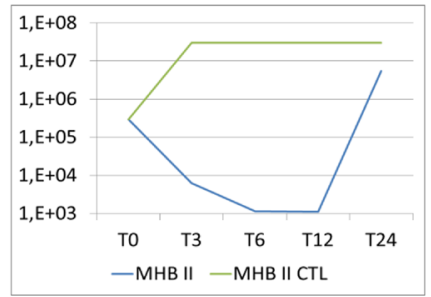

AgSulfad $(\mathrm{MIC}=13.5 ; \mathrm{MBC}=13.5)$

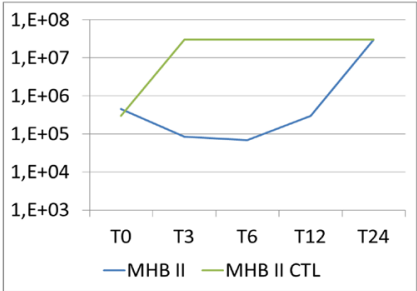

Chitosan $(\mathrm{MIC}=6.7 ; \mathrm{MBC}=6.7)$

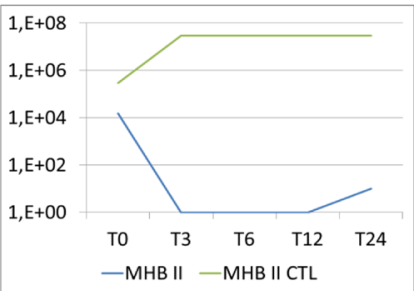

AgNitrate $(\mathrm{MIC}=6.7 ; \mathrm{MBC}=6.7)$

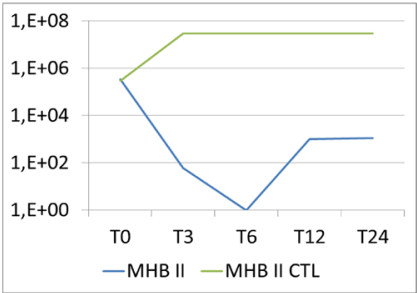

\section{Carbapenem-susceptible Enterobacter aerogenes}

Citrate $(\mathrm{MIC}=3.4 ; \mathrm{MBC}=3.4)$

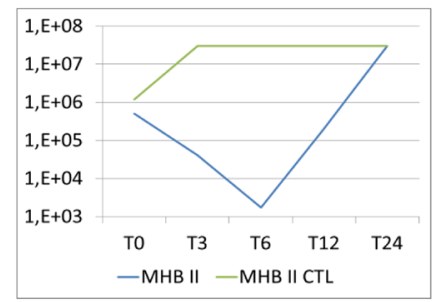

AgSulfad $(\mathrm{MIC}=13.5 ; \mathrm{MBC}=13.5)$

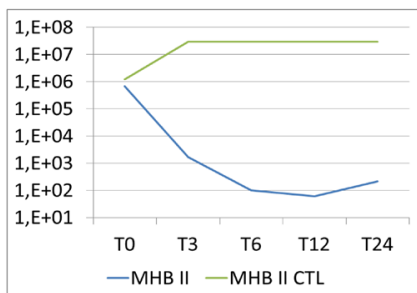

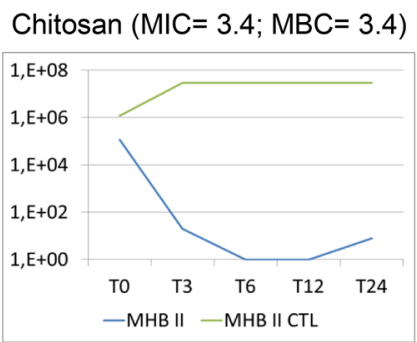

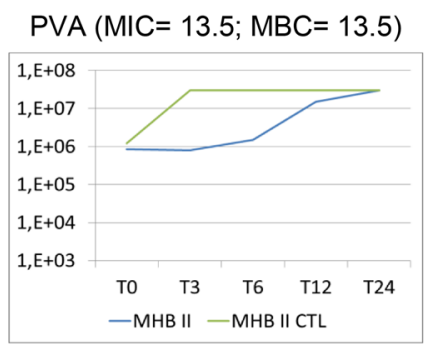

AgNitrate $(\mathrm{MIC}=3.4 ; \mathrm{MBC}=6.7)$

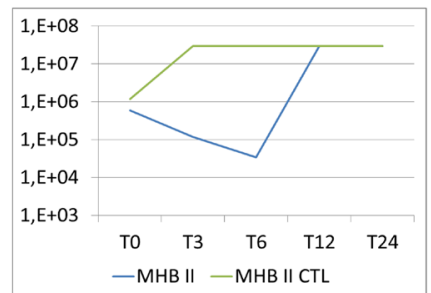

Fig. 9 Comparison of time-kill curves for a carbapenem-resistant K. pneumoniae (KPC) isolate and an isolate of carbapenem-susceptible $E$. aerogenes, using AgNPs particles (citrate, chitosan and PVA) and controls (silver sulfadiazine and silver nitrate). MHB II- Mueller Hinton Broth cation adjusted and microorganism; MHB II CTL-control without microorganisms. For commercial AgNPs control the curve was not done due to high MICs $\left(\mathrm{MIC} \geq 10 \mu \mathrm{g} \mathrm{mL}^{-1} ; \mathrm{MBC} \geq 10 \mu \mathrm{g} \mathrm{mL}^{-1}\right)$

showed by a mapping analysis using the X-ray energy dispersive spectrometer (EDS) the effect of AgNPs and pure ionic silver against $E$. coli isolates [4]. The silver AgNPs were well distributed through the isolate electrostatically adhered over the cellular membrane and inside the

\section{PVA $(M I C=13.5 ; M B C \geq 54)$}

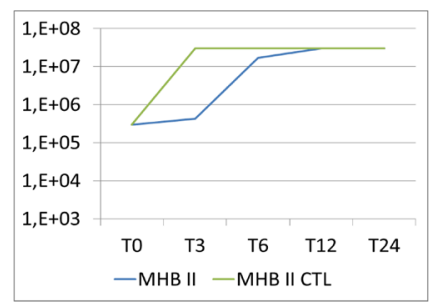


stability of the system PVA AgNPs as demonstrated by XRD (Fig. 3) and/or interaction with the $-\mathrm{OH}$ groups of PVA [34].

No impurities were detected in the XRD profile for AgNPs-PVA. However, some others peaks at $2 \theta$ angles of $32.2^{\circ}, 46.3^{\circ}, 54.7^{\circ}$ and $57.3^{\circ}$ were observed in the XRD profile for AgNPs-chitosan, which have been attributed in literature to both crystalline organic phase and $\mathrm{Ag}_{2} \mathrm{O}$ residues. Moreover, the spectrum of AgNPs-citrate shows no peak associate to metallic silver, which suggests that the metallic silver were oxidized to ions during the drying process. Together, these results provide evidence that the excellent in vitro inhibitory effect of citrate AgNPs and chitosan AgNPs against susceptible and MDR bacteria probably arises from the rapid oxidation process of these particles and release of $\mathrm{Ag}^{+}$ions and/or the high surface charge of the particles.

Several factors can have impact on the effect of AgNPs against microorganisms such as size, shape, stability and concentration of AgNPs [35]. Apparently the charge of AgNPs causes less interference over the effect. At biological $\mathrm{pH}$ values, the overall surface of the bacteria is negatively charged due to the dissociation of an excess number of carboxylic and other groups in the membrane [4]. Some studies reported that electrostatic attraction between negatively charged bacterial cells and positively charged AgNPs is essential for the activity of AgNPs [36]. However, our results for positive charged chitosan AgNP and negative citrate AgNP were equivalent. Sondi and Salopek-sondi showed that negatively charged AgNPs present excellent antibacterial activity against gram negative E. coli. [37]. According to the authors, negative AgNPs somehow interact with the bacterial membrane, causing structural changes and degradation [37]. Furthermore, several studies have demonstrated that the antibacterial activity of chitosan can result in a synergic effect in the Chitosan-AgNPs system [38, 39].

Regardless of the AgNPs charge, the growth inhibition of bacteria is related to the formation of $\mathrm{Ag}^{+}$from the surface of the nanoparticles, attacking the membrane lipids and leading to a breakdown of membrane function [40]. In this way, the antibacterial activities of AgNPs are critically dependent on surface oxidation and optimal particle dispersion [41]. Kim et al. demonstrated that the antioxidant $\mathrm{N}$-acetyl cysteine (NAC) could influence antimicrobial activity induced by a slightly negative charged AgNP (surface zeta potential of $-0.33 \mathrm{mV}$ ) [42]. The inhibitory effect was abolished by the addition of NAC, while NAC alone did not affect the antimicrobial activity. This can be explained by the fact that the nanosize allowed expansion of the contact surface of $\mathrm{Ag}^{+}$with the microorganisms despite the negative AgNP charge [42].
Studies had demonstrated the in vitro activity of AgNPs against Gram-negative and Gram-positive isolates, however the AgNPs activity against Gram-negative is not fully understood $[4,37]$. The AgNPs effect against Gram-negative could differ based on the in vitro method used, and few studies used the gold standard method by muramic acid release and quantification by a gas chromatography-mass spectrometry (GC-MS) $[8,10]$. Dawy et al. described better AgNPs inhibitory effect against $S$. aureus than E. coli and P. aeruginosa using agar diffusion method, arguing that the lipopolysaccharide (LPS) could have a protective effect in Gram-negative bacteria [40]. On the other hand, Shrivastava et al. evaluated by agar dilution and growth curve methods two strains of antimicrobial-susceptible microorganisms: $S$. aureus (ATCC 25923) and E. coli (ATCC 25922); and two antimicrobial resistant: E. coli and S. typhus, and showed better activity of AgNPs against Gram-negative [28]. Sondi and Salopek-Sondi studied one prototype of E. coli by agar dilution and growth curve and defended that Gramnegative bacteria are more susceptible to AgNPs because the positive charges of $\mathrm{Ag}^{+}$interact with the LPS of the cell membrane with greater affinity when compared to Gram-positive, resulting in the cell membrane pores [37]. Few studies used MIC and or MBC to evaluate the effect of AgNPs against bacteria [11, 43]. Egger et al. evaluated the effect of silica AgNPs and silver nitrate against a small number of Gram-negative $(\mathrm{n}=5)$ and Gram-positive $(\mathrm{n}=4)$ bacteria [43]. The MIC and MBC of these two groups differed significantly with greater susceptibility against Gram-negative. The authors speculating that this effect can be due to the peculiarities of the cell wall of bacteria, Gram-positive contain multiple layers of peptidoglycan (around $30 \mathrm{~nm}$ ) compared with the wall of Gram-negative bacteria (around $3 \mathrm{~nm}$ ). Another possibility is the high content of teichoic and lipoteichoic acids common in Gram-positive that have strong negative charge that may hijack free $\mathrm{Ag}^{+}$ions. Our $\mathrm{MIC}_{50}$ results against MDR $P$. aeruginosa were 3.37, 6.75 and $13.5 \mu \mathrm{g} / \mathrm{ml}$ respectively for Citrate AgNPs, chitosan and PVA lower than previously reported. Singh et al. using "green synthesized" AgNPs by Phyllanthus amarus described $\mathrm{MIC}_{50}$ of $6.25 \mu \mathrm{g} / \mathrm{ml}$ [11] and using Tinospora cordifoliapara $\mathrm{MIC}_{50}$ of $50 \mu \mathrm{g} / \mathrm{ml}$ against $P$. aeruginosa [44]. Ansari et al. studied the action of AgNPs against $S$. aureus and reported $\mathrm{MIC}_{90}$ for MSSA of $25 \mu \mathrm{g} / \mathrm{ml}$ and MRSA of $50 \mu \mathrm{g} / \mathrm{ml}$ [5]. These findings were higher than our results that showed $\mathrm{MIC}_{90}$ for MSSA/MRSA 13.5 and $6.75 \mu \mathrm{g} / \mathrm{ml}$ respectively for Citrate and chitosan AgNPs. Thus, this issue is still controversy and more studies are need.

Our results showed the great potential of silver AgNPs for clinical use. However, its toxicity and the concern 
with the risk to the environment, with the growing accumulation of these substances when disposed should be addressed.

\section{Conclusions}

The synthesized AgNPs, especially citrate and chitosan AgNPs, showed excellent in vitro inhibitory effect against susceptible and MDR bacteria. The AgNPs effect was superior against Gram-negative compared to Gram-positive. The inhibitory effect (MIC) was similar against MDR and susceptible bacteria, whit bactericidal (MBC) effect higher against susceptible isolates. It seems that media supplement with blood should be avoided to evaluate in vitro activity of AgNPs. Diffusion method in depth can be used as screening test, and MBC/MIC and time kill as reference methods.

The bactericidal effect of AgNPs can be translated into important therapeutic and clinical options in the future, especially considering the shortage of new antimicrobials against the emerging antimicrobial resistant microorganisms, in particular against Gram-negative bacteria.

\section{Methods}

The study was performed in the Laboratory of Medical Research 54 (LIM-54) of the Faculty of Medicine of the University of São Paulo (FM-USP) and the Institute of research and development of Laboratório Fleury Medicina e Saúde.

\section{Microorganisms}

Silver compounds were studied against a set of 90 clinical microorganisms, including MDR $(\mathrm{n}=54)$ and susceptible microorganisms $(n=36)$. The MDR belonged to different clones, previously characterized by pulsed field gel electrophoresis (PFGE), and obtained from laboratory strain bank LIM-54. Susceptible strains from Laboratório Alerta (Universidade Federal de São Paulo, UNIFESP) were also included and The American Type Culture Collection (ATCC) and the Instituto Nacional de Controle de Qualidade em Saúde (INCQS)were used as control. The following resistance genes had been previously studied in Gram-negative and Gram-positive bacteria: carbapenem and polymyxin B-resistant $A$. baumannii (harboring $o x a-{ }_{23}$ and $o x a-{ }_{143}$ genes) $(\mathrm{n}=12)$, carbapenem and polymyxin B-susceptible $A$. baumannii $(\mathrm{n}=5)$, carbapenemresistant $P$. aeruginosa (harboring SPM and VIM genes) $(\mathrm{n}=5)$, carbapenem-susceptible $P$. aeruginosa $(\mathrm{n}=8)$, carbapenem-resistant Enterobacteriaceae (harboring extending spectrum beta lactamase ESBL and KPC-2 genes) $(n=12)$, carbapenem-susceptible Enterobacteriaceae $(\mathrm{n}=9)$ and sulfamethoxazole/trimethoprim- and levofloxacin-resistant S. maltophilia (harboring Sul-1 and Sul- $_{2}$ genes) $(\mathrm{n}=2)$. Oxacillin-resistant $S$. aureus (methicillin resistant $S$. aureus MRSA-SCCmec I, II, III, IVa, IVb and IVc positives) $(\mathrm{n}=5)$, oxacilin-susceptible S. aureus $(\mathrm{n}=8)$, vancomycin-resistant Enterococcus spp. (harboring vanA gene) $(\mathrm{n}=8)$, vancomycin-susceptible Enterococcus spp. $(\mathrm{n}=6)$

\section{AgNPs}

The AgNPs particles (whit citrate, PVA and chitosan) were produced in the Instituto de Física de São Carlos, Universidade de São Paulo (IFSC-USP). All AgNPs were produced from $1 \mathrm{mM}$ of $\mathrm{AgNO}_{3}$ (Sigma-Aldrich, St. Louis, MO, USA), resulting in $108 \mu \mathrm{g} \mathrm{mL}^{-1}$ AgNPs.

The amount of capping agents (citrate, chitosan and PVA) used in each synthesis depended on the stabilizing agent and it was optimized in order to have the best stability. Since citrate, PVA and chitosan presents different molecular weight and functionalization mechanism, the capabilities of them to stabilize the silver nanoparticles are different and the ratios between silver and capping agent had to be optimized in each case.

It is worth emphasizing that the main idea of this study was to evaluate which system could present the best antimicrobial activity in terms of surface charge, size, and stability. Since these capping agents are very studied in synthesis of silver nanoparticles and present significant differences, they were chosen for this comparative study.

\section{Synthesis of PVA, chitosan and citrate AgNPs PVA AgNPS}

PVA AgNPs were synthesized based on modification of previous methods $[45,47,48]$. Briefly, $30 \mathrm{~mL}$ of a $4 \mathrm{~g} \mathrm{~L}^{-1}$ solution of polyvinyl alcohol (PVA, Mw 89,000-98,000) (Sigma-Aldrich's. Louis, MO, USA) were mixture to $30 \mathrm{~mL}$ of $1 \mathrm{mM}$ of $\mathrm{AgNO}_{3}$ (Sigma-Aldrich) at room temperature and under magnetic stirring. After $10 \mathrm{~min}, 1 \mathrm{~mL}$ of a cold solution of $0.1 \mathrm{~mol} \mathrm{~L}^{-1} \mathrm{NaBH}_{4}$ (Sigma-Aldrich, St. Louis, MO, USA) were added. The system has passed from transparent color to yellow, indicating the formation of the nanoparticles [47].

\section{Chitosan AgNPs}

A similar strategy was used to produce the chitosan AgNPs [49]. Briefly, $30 \mathrm{~mL}$ of a $1 \mathrm{~g} \mathrm{~L}^{-1}$ chitosan solution (Medium molecular weight, Sigma-Aldrich, St. Louis, MO, USA) containing $1 \%$ acetic acid were added to $30 \mathrm{~mL}$ of a $1 \mathrm{mM}$ solution of $\mathrm{AgNO}_{3}$ (Sigma-Aldrich, St. Louis, MO, USA) at room temperature and under magnetic stirring, followed by adding $1 \mathrm{~mL}$ of $0.1 \mathrm{~mol}$ $\mathrm{L}^{-1} \mathrm{NaBH}_{4}$ cold solution. The color of the system also changed from transparent to yellow. 


\section{Citrate AgNPs}

Citrate AgNPs were synthesized based on previous methods with modifications [46]. For this, $30 \mathrm{~mL}$ of $1 \mathrm{mM}$ $\mathrm{AgNO}_{3}$ solution (Sigma-Aldrich) were heated to boiling in a round-bottom flask connected to a condenser. After reaching the boiling point, $13 \mathrm{~mL}$ of a sodium citrate (Sigma-Aldrich) solution of $1 \% \mathrm{w} / \mathrm{v}$ were added under vigorous agitation. After a few minutes, the solution color changed from transparent to yellow and the heating system was switched off.

\section{Characterization of AgNPs (PVA, chitosan and citrate)}

The optical properties of the AgNPs were characterized by Ultraviolet-Visible spectroscopy (UV-VIS) (HITACHI, U-2900) using a $1 \mathrm{~cm}$ quartz cell. The size and morphology of the silver nanoparticles were analyzed by Field Emission Gun Scanning Electron MicroscopeFEG-SEM (Zeiss) at $2.0 \mathrm{kV}$. For this, diluted samples of PVA, chitosan and citrate stabilized AgNPs were deposited in silicon substrates without any further coating or processing and dried at room temperature. The surface charge of the particles was evaluated in suspension by zeta potential measurements (Zetasizer Nano, ZS90). The crystal structure of silver nanoparticles stabilized by PVA, chitosan and citrate were studied with X-ray powder diffraction (XRD) in a Rigaku diffractometer, with CuKa $(1.5406 \AA)$ radiation. For this, the particles were synthesized according to the described previously, centrifuged to remove the excess of stabilizers and dried at room temperature.

\section{Controls}

Silver sulfadiazine (Sigma-Aldrich, St. Louis, MO, USA) and silver nitrate (Sigma-Aldrich, St. Louis, MO, USA) at initial concentration of $54 \mu \mathrm{g} \mathrm{mL}^{-1}$ were used as control.

The commercial Sigma-Aldrich AgNPs (St. Louis, MO, USA) at concentration of $20 \mu \mathrm{g} \mathrm{mL}^{-1}$ with $60 \mathrm{~nm}$ sized nanoparticles, stabilized by citrate was used as control.

Tests The aim of the selected methods was to study the antibacterial effect of AgNPs produced. Their inhibitory and bacteriostatic/bactericide effect, as well as the dynamic of bacterial killing were evaluated using the following tests:

\section{Growth inhibition by diffusion}

The test was carried out according to the agar well diffusion (AWD) method $[12,13]$ as a screening of AgNPs inhibitory effect. To evaluate possible interference of enriched culture medium the growth inhibition tests in depth were performed using Mueller-Hinton AgarMHA (Biomerieux, Marcy L'Etoile, France) and MHA with sheep blood $5 \%$ (Difco, Sparks, MD, USA).
The holes made in agar with $5 \mathrm{~mm}$ diameter were aseptically filled with $50 \mu \mathrm{L}$ of AgNPs and controls. The plates were then incubated at $36 \pm 1{ }^{\circ} \mathrm{C}$ for $24 \mathrm{~h}$ and inhibition zones measured, in millimeters, under reflected light.

\section{Determination of minimum inhibitory concentration (MIC)}

The AgNPs inhibitory power against bacteria was evaluated using MIC. The MIC determination was carried out with Mueller-Hinton Broth -MHB II and Trypticase Soy Broth-TSB, and both showed similar results. The impact of blood was evaluated using MHB II $1.25 \%$ sheep blood. The results were affected by the presence of blood, thus MHBII was chosen to perform all the tests.

The samples were diluted in a serial logarithmic base $2\left(\log _{2}\right)$, in MHB cation adjusted (MHB II) (BBL, Sparks, MD, USA), plus microbial suspensions with $10^{4} \mathrm{cfu}$ in MHB II (Difco), resulting in a micro dilution panel from $1: 4$ to $1: 256$, following the methodology of CLSI document M07-A9 [14]. According to original concentration, after initial 1:4 dilution PVA, chitosan and citrate AgNPs started from $27 \mu \mathrm{g} \mathrm{mL}^{-1}$, the controls silver nitrate and silver sulfadiazine started from $13.5 \mu \mathrm{gmL}^{-1}$ and commercial AgNPs from $5 \mu \mathrm{g} \mathrm{mL}{ }^{-1}$. The 96 micro well plates were incubated under $35 \pm 1{ }^{\circ} \mathrm{Cfor} 16$ to $18 \mathrm{~h}$ and read visually with transmitted light observing the presence or absence of turbidity. The first well with no microbial growth was defined as the MIC, expressed in $\mu \mathrm{g} \mathrm{mL} L^{-1}$. To evaluate the reproducibility of the method, some tests were carried out in triplicate for sampling.

Minimum bactericidal concentration determination (MBC) The concentration of AgNPs required to achieve the bactericidal effect was defined using MBC. After reading the MIC, all wells without visible turbidity were plated in MHA and incubated under $35 \pm 1{ }^{\circ} \mathrm{C}$ for $16-18 \mathrm{~h}$. The plates were read visually with reflected light, observing the presence or absence of macroscopic bacterial growth. The lower dilution without macroscopic bacterial growth was defined as MBC. According to CLSI method for antimicrobial drugs, described in the document M7-A9 [14].

The MBC/MIC ratio was used to define the mode of activity of silver derivatives: bactericidal when scores are 1,2 and 4 or bacteriostatic if score $>4$ [26].

\section{Time-kill tests}

The dynamic of the AgNPs inhibitory effect was carried out using the time-kill curves under AgNPs concentration equal to $1 \times$ MIC [50]. Four representative strains of MDR and antimicrobial susceptible Gram-positive and Gram-negative were evaluated by this method, one strain of $S$. aureus oxacilin-resistant and one oxacilinsusceptible, $K$. pneumonia carbapenem-resistant and $E$. 
aerogenes carbapenem-susceptible evaluated according with previous described $[51,52]$. Shortly, after know the MIC for each AgNPs and microorganism evaluated were made tubes with $10 \mathrm{ml}$ of MHBII and AgNPs at 1xMIC and $10^{5} \mathrm{cfu} / \mathrm{mL}$ of each microorganism. For the negative control, AgNPs were substituted for MHBII. All tubes were incubated under $36 \pm 1{ }^{\circ} \mathrm{C}$ and the number of viable microorganisms quantified on MHA at time $0,3,6,12$ and $24 \mathrm{~h}$. The number of viable cfu in each time was plotted on a graph profiling the time-kill curve, compared to the curve of positive and negative controls.

The time-kill was not performed for the isolates with indeterminate $\mathrm{MIC}$, that is, above the highest concentration tested $\left(\geq 54 \mu \mathrm{g} \mathrm{mL}^{-1}, \geq 27 \mu \mathrm{g} \mathrm{mL}^{-1}\right.$ $\mathrm{e} \geq 10 \mu \mathrm{g} \mathrm{mL}^{-1}$ ). Except for MRSA isolate against the commercial AgNPs and MSSA isolate against PVA and commercial AgNPs. These were deliberately tested to show the shape of the curves in sub-inhibitory conditions.

To evaluate the blood interference observed in agar diffusion and MIC, for the isolated S. aureus MRSA (M01) the curve was held in MHBII broth and MHBII $1.25 \%$ sheep blood broth.

\begin{abstract}
Abbreviations
AgNPs: silver nanoparticles; ATCC: American Type Culture Collection; CLSI: Clinical and Laboratory Standards Institute; DLS: dynamic light scattering; EDS: energy dispersive spectrometer; ESBL: extended spectrum beta lactamase; FM-USP: Faculty of Medicine of the University of São Paulo; GC-MS: gas chromatography-mass spectrometry; INCQS: Instituto Nacional de Controle de Qualidade em Saúde; ISO: International Organization for Standardization; KPC: Klebsiella pneumoniae carbapenemase; LIM: Laboratory of Medical Research; LPS: pipopolysaccharide; MBC: minimum bactericidal concentration; MDR: multidrug resistant microorganisms; MHA: mueller-hinton agar; MHB: mueller hinton broth; MIC: minimum inhibitory concentration; MRSA: methicillin resistant Staphylococcus aureus; MSSA: methicillin susceptible S. aureus; NAC: $n$-acetyl cysteine; PFGE: pulsed field gel electrophoresis; PVA: polyvinyl alcohol; SCC-mec: staphylococcal cassette chromosome mec; SPM: São Paulo metallo-beta-lactamase; TSB: triptcase soy broth; UNIFESP: Universidade Federal de São Paulo; USP: Universidade de São Paulo; VIM: Verona imipenemase; VRE: vancomycin resistant Enterococcus; XRD: X-ray diffraction.
\end{abstract}

\begin{abstract}
Authors' contributions
EDC, FFF and VSM have made substantial contributions to conception and design; acquisition, analysis and interpretation of data; have been involved in drafting the manuscript critically for important intellectual content. LFPF, JPO, MMS and RAO have made substantial contributions to conception and design. VZ have made substantial contributions to conception and design, analysis and interpretation of data; have been involved in revising the manuscript critically for important intellectual content. ASSL and SFC have made substantial contributions to analysis and interpretation of data, have been involved in drafting and revising the manuscript critically for important intellectual content and have given final approval of the version to be published, All authors read and approved the final manuscript, except LFPF (in memorian).
\end{abstract}

\footnotetext{
Author details

${ }^{1}$ Department of Infectious Diseases, University of São Paulo, São Paulo, Brazil. ${ }^{2}$ Department of Surgery São Paulo, University of São Paulo, São Paulo, Brazil.

${ }^{3}$ Faculty of Engenier São Paulo, University of São Paulo, São Paulo, Brazil.

${ }^{4}$ Nanomedicine and Nanotoxicology Group, University of São Paulo, IFSC, São Paulo, Brazil. ${ }^{5}$ LIM-54 (Laboratório de Bacteriologia), Instituto de Medicina
}

Tropical, Av. Dr. Enéas de Carvalho Aguiar, 470, Prédio II, $1^{\circ}$ andar, Sala 112, Cerqueira César, São Paulo, SP 054503-00, Brazil.

\section{Compliance with ethical guidelines}

\section{Competing interests}

The authors declare that they have no competing interest.

\section{Approval by the research ethics committee}

This study was reviewed and approved by the Research Ethics Committee of FM-USP on December 15th, 2010 (research protocol number 378/10).

\section{Funding}

This study was supported by a Grant from the Coordenação de Aperfeiçoamento de Pessoal de Nível Superior (CAPES) and Conselho Nacional de Desenvolvimento Científico e Tecnológico (CNPq) awarded to Nanobiotec Project number1290/2009.

Received: 29 September 2014 Accepted: 9 September 2015 Published online: 05 October 2015

\section{References}

1. Planquette B, Timsit JF, Misset BY, Schwebel C, Azoulay E, Adrie C, Vesin A, Jamali S, Zahar JR, Allaouchiche B, et al. Pseudomonas aeruginosa ventilator-associated pneumonia predictive factors of treatment failure. Am J Respir Crit Care Med. 2013;188:69-76.

2. Webb GF, D'Agata EMC, Magal P, Ruan S. A model of anti bioticresistant bacterial epidemics in hospitals. Proc Natl Acad Sci USA. 2005;102:13343-8.

3. Lara HH, Ayala-Nunez NV, Turrent LDI, Padilla CR. Bactericidal effect of silver nanoparticles against multidrug-resistant bacteria. World J Microbiol Biotechnol. 2010;26:615-21.

4. Morones JR, Elechiguerra JL, Camacho A, Holt K, Kouri JB, Ramirez JT, Yacaman MJ. The bactericidal effect of silver nanoparticles. Nanotechnology. 2005; 16:2346-53.

5. Ansari MA, Khan HM, Khan AA, Malik A, Sultan A, Shahid M, Shujatullah F, Azam A. Evaluation of antibacterial activity of silver nanoparticles against MSSA and MRSA on isolates from skin infections. Biol Med. 2011;3:141-6.

6. Gong P, Li H, He X, Wang K, Hu J, Tan W, Tan S, Zhang XY. Preparation and antibacterial activity of $\mathrm{Fe}_{3} \mathrm{O}_{4} @ A g$ nanoparticles. Nanotechnology. 2007;18:604-11.

7. Chaloupka K, Malam Y, Seifalian AM. Nanosilver as a new generation of nanoproduct in biomedical applications. Trends Biotechnol. 2010;28:580-8.

8. ISO/TS16550:2014: Nanotechnologies_determination of silver nanoparticles potency by release of muramic acid from Staphylococcus aureus.

9. ISO/TR16197:2014: Nanotechnologies—compilation and description of toxicological screening methods for manufactured nanomaterials.

10. Mirzajani F, Ghassempour A, Aliahmadi A, Esmaeili MA. Antibacterial effect of silver nanoparticles on Staphylococcus aureus. Res Microbiol. 2011;162:542-9.

11. Singh K, Panghal M, Kadyan S, Chaudhary U, Yadav JP. Green silver nanoparticles of Phyllanthus amarus: as an antibacterial agent against multi drug resistant clinical isolates of Pseudomonas aeruginosa. J Nanobiotechnol. 2014;12:40.

12. Kalishwaralal K, BarathManiKanth S, Pandian SRK, Deepak V, Gurunathan S. Silver nanoparticles impede the biofilm formation by Pseudomonas aeruginosa and Staphylococcus epidermidis. Colloids Surf B-Biointerf. 2010;79:340-4.

13. Pirnay JP, De Vos D, Cochez C, Bilocq F, Pirson J, Struelens M, Duinslaeger L, Cornelis P, Zizi M, Vanderkelen A. Molecular epidemiology of Pseudomonas aeruginosa colonization in a burn unit: persistence of a multidrug-resistant clone and a silver sulfadiazine-resistant clone. J Clin Microbiol. 2003;41:1192-202.

14. Methods for Dilution Antimicrobial Susceptibility Tests for Bacteria That Grow Aerobically; Approved Standard-Ninth Edition. CLSI document M07-A9 (ISBN 1-56238-783-9 [Print]; ISBN 1-56238-784-7 [Electronic]). In Clinical and Laboratory Standards Institute. 950 West Valley Road, Suite 
2500, Wayne, Pennsylvania 19087, USA: Clinical and Laboratory Standards Institute; 2012.

15. Pileni MP. Nanosized particles made in colloidal assemblies. Langmuir. 1997;13:3266-76

16. Solomon SD, Bahadory M, Jeyarajasingam AV, Rutkowsky SA, Boritz C, Mulfinger L. Synthesis and study of silver nanoparticles. J Chem Educ. 2007;84:322-5.

17. Sharma VK, Yngard RA, Lin Y. Silver nanoparticles: green synthesis and their antimicrobial activities. Adv Colloid Interface Sci. 2009;145:83-96.

18. Coronado EA, Encina ER, Stefani FD. Optical properties of metallic nanoparticles: manipulating light, heat and forces at the nanoscale. Nanoscale. 2011;3:4042-59.

19. Ruparelia JP, Chatteriee AK, Duttagupta SP, Mukherji S. Strain specificity in antimicrobial activity of silver and copper nanoparticles. Acta Biomater. 2008:4:707-16.

20. Zhang JT, Li XL, Sun XM, Li YD. Surface enhanced Raman scattering effects of silver colloids with different shapes. J Phys Chem B. 2005;109:12544-8.

21. Shameli K, Bin Ahmad M, Yunus W, Rustaiyan A, Ibrahim NA, Zargar M, Abdollahi Y. Green synthesis of silver/montmorillonite/chitosan bionanocomposites using the UV irradiation method and evaluation of antibacterial activity. Int J Nanomed. 2010;5:875-87.

22. Philip D. Biosynthesis of Au, Ag and Au-Ag nanoparticles using edible mushroom extract. Spectrochimica Acta Part a Mol Biomol Spectrosc. 2009;73:374-81.

23. Shankar SS, Ahmad A, Sastry M. Geranium leaf assisted biosynthesis of silver nanoparticles. Biotechnol Prog. 2003;19:1627-31.

24. Wang HS, Qiao XL, Chen JG, Ding SY. Preparation of silver nanoparticles by chemical reduction method. Colloids Surf a-Physicochem Eng Asp. 2005;256:111-5.

25. Stebounova LV, Adamcakova-Dodd A, Kim JS, Park H, O'Shaughnessy PT, Grassian VH, Thorne PS. Nanosilver induces minimal lung toxicity or inflammation in a subacute murine inhalation model. Particle Fibre Toxicol 2011;8

26. Ayala-Núñez NV, Villegas HL, Turrent LC, Padilla CR. Silver nanoparticles toxicity and bactericidal effect against methicillin-resistant Staphylococcus aureus : nanoscale Does Matter. NanoBiotechnology. 2009;5:2-9.

27. Jena P, Mohanty S, Mallick R, Jacob B, Sonawane A. Toxicity and antibacterial assessment of chitosan-coated silver nanoparticles on human pathogens and macrophage cells. Int J Nanomed. 2012;7:1805-18.

28. Shrivastava S, Bera T, Roy A, Singh G, Ramachandrarao P, Dash D. Characterization of enhanced antibacterial effects of novel silver nanoparticles. Nanotechnology 2007; 18.

29. Patil RS, Kokate MR, Jambhale CL, Pawar SM, Han SH, Kolekar SS. One-pot synthesis of PVA-capped silver nanoparticles their characterization and biomedical application. Adv Nat Sci Nanosci Nanotechnol. 2012;3:015013.

30. Nowack B, Krug HF, Height M. 120 years of nanosilver history: implications for policy makers. Environ Sci Technol. 2011;45:1177-83.

31. Schacht VJ, Neumann LV, Sandhi SK, Chen L, Henning T, Klar PJ, Theophel K, Schnell S, Bunge M. Effects of silver nanoparticles on microbial growth dynamics. J Appl Microbiol. 2013;114:25-35.

32. Rai M, Yadav A, Gade A. Silver nanoparticles as a new generation of antimicrobials. Biotechnol Adv. 2009;27:76-83.

33. Zhao GJ, Stevens SE. Multiple parameters for the comprehensive evaluation of the susceptibility of Escherichia coli to the silver ion. Biometals. 1998;11:27-32.

34. Loo CY, Young PM, Lee WH, Cavaliere R, Whitchurch CB, Rohanizadeh R. Non-cytotoxic silver nanoparticle-polyvinyl alcohol hydrogels with antibiofilm activity: designed as coatings for endotracheal tube materials. Biofouling. 2014;30:773-88.

35. Rai MK, Deshmukh SD, Ingle AP, Gade AK. Silver nanoparticles: the powerful nanoweapon against multidrug-resistant bacteria. J Appl Microbiol. 2012;112:841-52.

36. Stoimenov PK, Klinger RL, Marchin GL, Klabunde KJ. Metal oxide nanoparticles as bactericidal agents. Langmuir. 2002;18:6679-86.

37. Sondi I, Salopek-Sondi B. Silver nanoparticles as antimicrobial agent: a case study on E-coli as a model for Gram-negative bacteria. J Colloid Interface Sci. 2004;275:177-82.
38. Benhabiles S, Salah R, Lounici H, Drouiche N, Goosen MFA, Mameri N. Antibacterial activity of chitin, chitosan and its oligomers prepared from shrimp shell waste. Food Hydrocolloids. 2012;29:48-56.

39. Sanpui P, Murugadoss A, Prasad PVD, Ghosh SS, Chattopadhyay A. The antibacterial properties of a novel chitosan-Ag-nanoparticle composite. Int J Food Microbiol. 2008;124:142-6.

40. Dawy M, Rifaat HM, Moustafa SA, Mousa HA. Physicochemical studies on nano silver particles preparated by different techniques. Aust J Basic Appl Sci. 2012;6:257-62.

41. Lok CN, Ho CM, Chen R, He QY, Yu WY, Sun H, Tam PKH, Chiu JF, Che CM. Silver nanoparticles: partial oxidation and antibacterial activities. J Biol Inorg Chem. 2007;12:527-34.

42. Kim JS, Kuk E, Yu KN, Kim JH, Park SJ, Lee HJ, Kim SH, Park YK, Park YH, Hwang $C Y$, et al. Antimicrobial effects of silver nanoparticles. Nanomed Nanotechnol Biol Med. 2007;3:95-101.

43. Egger S, Lehmann RP, Height MJ, Loessner MJ, Schuppler M. Antimicrobial properties of a novel silver-silica nanocomposite material. Appl Environ Microbiol. 2009:75:2973-6.

44. Singh K, Panghal M, Kadyan S, Chaudhary U, Yadav JP. Antibacterial activity of synthesized silver nanoparticles from Tinospora cordifolia against multi drug resistant strains of Pseudomonas aeruginosa isolated from burn patients. J Nanomed Nanotechnol. 2014;5:192.

45. Khanna PK, Singh N, Charan S, Subbarao V, Gokhale R, Mulik UP. Synthesis and characterization of Ag/PVA nanocomposite by chemical reduction method. Mater Chem Phys. 2005;93:117-21.

46. Lee PC, Meisel D. Adsorption and surface-enhanced Raman of dyes on silver and gold sols. J Phys Chem. 1982;86:3391-5.

47. Temgire MK, Joshi SS. Optical and structural studies of silver nanoparticles. Radiat Phys Chem. 2004;71:1039-44.

48. Cao XL, Cheng C, Ma YL, Zhao CS. Preparation of silver nanoparticles with antimicrobial activities and the researches of their biocompatibilities. J Mat Sci Mat Med. 2010;21:2861-8.

49. Shih CM, Shieh YT, Twu YK. Preparation of gold nanopowders and nanoparticles using chitosan suspensions. Carbohydr Polym. 2009;78:309-15.

50. Hindler J. Tests to assess bactericidal activity. In: Isenberg HD, editor. Clinical microbiology procedures handbook. Washington, D.C.: American Society for Microbiology; 1992.

51. Leite AM, Lima EO, Souza EL, Diniz MF, Trajanoll VN, Medeiros IA. Inhibitory effect of b-pinene, a-pinene and eugenol on the growth of potential infectious endocarditis causing Gram-positive bacteria. Revista Brasileira de Ciências Farmacêuticas. 2007;43:121-6.

52. GiamarellosBouroulis EJ, Grecka P, Giamarellou H. Comparative in vitro interactions of ceftazidime, meropenem, and imipenem with amikacin on multiresistant Pseudomonas aeruginosa. Diagn Microbiol Infect Dis. 1997;29:81-6

\section{Submit your next manuscript to BioMed Central and take full advantage of:}

- Convenient online submission

- Thorough peer review

- No space constraints or color figure charges

- Immediate publication on acceptance

- Inclusion in PubMed, CAS, Scopus and Google Scholar

- Research which is freely available for redistribution

Submit your manuscript at

www.biomedcentral.com/submit

C Biomed Central 